\title{
Near-Optimal Watermark Estimation and Its Countermeasure: Antidisclosure Watermark for Multiple Watermark Embedding
}

\author{
Chun-Shien Lu, Member, IEEE, and Chao-Yung Hsu
}

\begin{abstract}
Robustness is a critical requirement for a watermarking scheme to be practical. Current watermarking methods usually claim a certain degree of robustness against those attacks that aim to destroy a hidden watermark at the expense of degrading the quality of the media data. However, there exist watermarkestimation attack (WEA), such as the collusion attack, which can remove watermarks while making the attacked data further transparent to its original. Another kind of attack is the copy attack, which can cause protocol ambiguity within a watermarking system. The aim of this paper is to deal with the WEA, which is clever at disclosing hidden information for unauthorized purposes. To this end, we begin by gaining insight into the WEA, leading to formal definitions of optimal watermark estimation and near-perfect cover data recovery. Subject to these definitions, a new content-dependent watermark (CDW) is proposed to deal with watermark-estimation attack. Mathematical analyses and experimental results consistently verify the effectiveness of the proposed watermark estimation and recovery algorithm, and the proposed content-dependent watermark. To our knowledge, this anti-disclosure watermark is the first work that can give those multiple-redundant watermark embedding approaches resistance to both collusion and copy attacks.
\end{abstract}

Index Terms-Collusion attack, content-dependent watermark, copy attack, cover data recovery, media hash, robustness, watermark estimation.

\section{INTRODUCTION}

D ATA HIDING is the technology of embedding a piece of information into cover media data (also called "cover data" or "host data") for the purpose of carrying out specific missions. In this emerging field, digital watermarking and steganography are two main branches that have attracted a great amount of interest in the last decade [12], [13], [30], [33]. This paper will focus on digital watermarking, which has been popularly employed for copyright protection, content authentication, access control, and other purposes.

No matter what kinds of applications are considered, robustness is the critical issue affecting the practicability of a watermarking system. In data hiding, robustness refers to the capability of resistance to attacks that are used to destroy or remove hidden watermarks. As previously discussed in [43], attacks can be classified into four categories: 1) removal attacks; 2) geometrical attacks; 3) cryptographic attacks; and 4) protocol at-

Manuscript received May 8, 2006; revised September 25, 2006. This work was supported by the National Science Council under NSC Grant 93-2422-H001-004. This paper was recommended by Associate Editor Q. Sun.

The authors are with the Institute of Information Science, Academia Sinica, Taipei, Taiwan 115, R.O.C. (e-mail: lcs@iis.sinica.edu.tw; cyhsu@iis.sinica. edu.tw).

Digital Object Identifier 10.1109/TCSVT.2006.888837 tacks. The robustness of the current watermarking methods has been examined with respect to removal attacks or geometrical attacks or both. In particular, removal attacks contain operations, including filtering, compression, and noise adding, that more or less degrade the quality of the media data. While the employed removal attack cannot guarantee successful removal of the hidden watermark, the media quality will inevitably be reduced. However, there indeed exist attacks that can defeat a watermarking system without sacrificing media quality. Among the currently known attacks [43], the collusion attack [15], [35], [37], which is a removal attack, and the copy attack [3], [17], which is a protocol attack, are typical examples of attacks that can achieve the aforementioned goal. The common step used to realize a collusion or copy attack is watermark estimation, which is usually accomplished by means of a denoising procedure. Consequently, we call both the collusion attack and copy attack watermark-estimation attack (WEA).

The aim of the collusion attack is to collect and analyze a set of watermarked media data ${ }^{1}$ so that an unwatermarked copy can be constructed to create the false negative problem. In digital watermarking, a collusion attack naturally occurs in video watermarking because a video is composed of many frames, and one way of watermarking a video is to embed the same watermark into all the frames. This scenario was first addressed in [37]. However, we argue that the collusion attack is not exclusively applied to video watermarking. In the past few years, image watermarking with resistance to geometrical attacks has received much attention because even a slight geometrical distortion may disorder the hidden watermark bits and disable watermark detection. In view of this fact, some researches [4], [38], [44] inserted multiple redundant watermarks into an image in the hope that robustness could be maintained as long as at least one watermark existed. Commonly, various kinds of image units, such as meshes [4], disks [38], or blocks [44], are extracted as carriers for embedding. Taking advantage of this unique characteristic, we propose to treat each image unit in an image like a frame in a video; in this way, collusion attacks can be equally applied to those image watermarking methods that employ a multiple redundant watermark embedding strategy. Therefore, once the hidden watermarks are successfully removed by means of a collusion attack, the false negative problem occurs even though no geometrical attack

\footnotetext{
${ }^{1}$ This set of watermarked media data in fingerprinting [39], [40] is generated from the same cover data but individually embedded with different watermarks, while in watermarking, it is generated from visually similar/dissimilar image embedding units [4], [22], [38], [44] or video frames [37] embedded with the same watermark.
} 
is imposed on watermarked images. Of particular interest are possible fidelity improvements of attacked images as a result of a collusion attack. Hence, the collusion attack has a potential advantage over its counterpart, the removal attack.

In contrast to the collusion attack, the copy attack [17] has been developed to create the false positive problem; i.e., a situation in which one can successfully detect a watermark from unwatermarked data. The copy attack was first applied to image watermarking and is carried out as follows: 1) a watermark is first predicted from a watermarked image; 2 ) the predicted watermark is added into a target image to create a counterfeit watermarked image; and 3) from the counterfeit image, a watermark is detected that wrongly claims rightful ownership. Compared with the collusion attack, the copy attack is not restricted to execution on more than one media data; thus, it is more flexible. We will also show that the copy attack is rather easier to carry out than the denoising attack (a special case of the collusion attack). Based on the aforementioned reasons, the copy attack must be taken into consideration when the robustness of a watermarking system is to be evaluated.

In this paper, we propose a new content-dependent watermark, which is a hybrid of a media hash and an embedded signal, to cope with watermark-estimation attack (WEA). The related works [3], [8], [15], [37] proposed to resist either the collusion attack or the copy attack are briefly discussed as follows. In [3], Barr et al. proposed to concatenate a watermark message and a block signature as an embedded signal to combat the copy attack. Their block signature is generated by first choosing the $16 \times 16$ DCT coefficients in the lowest frequency subbands (the DC term is zeroed out) from a $128 \times 128$ DCT transformed image block. Then, the median value of the 256 coefficients is used as a threshold to yield a binary sequence, where each element is generated by comparing a coefficient with the threshold. Deguillaume et al. [8] proposed a hybrid watermark scheme joining a robust watermark and a fragile watermark to cope with the copy attack. That is, two watermarks that play different roles are embedded. Kirovski et al. [15] proposed a watermark-fingerprint system, where all copies of a protected object are identically watermarked using a same secret embedding key, but different detection keys are used to deal with the collusion attack. Basically, their detection key is a combination of the embedding key and a watermark carrier, which is different for different detectors. The watermark carrier is used to hide the detection key in that knowledge of the detection key does not lead to knowledge of the embedding key. In [37], Swanson et al. proposed the content-dependent watermark to solve the deadlock problem that leads to ambiguous rightful ownership. Their watermark is designed to be content-dependent by exploiting the provable security of a cryptographic one-way hash function. In this study, a new content-dependent watermark giving multiple-redundant watermark embedding approaches [4], [38], [44] the resistance to both the collusion and copy attacks is proposed. It is important to note that we present a different design strategy [21], where the embedded signal is a combination of a watermark message and a block media hash through shuffling, as will be described in Section IV. By comparing our method and those methods in [3], [8], [15], [37], it is obvious that either the design strategy of a content-dependent watermark or the employed application domain is different. These characteristics clearly distinguish our method from the existing methods. Moreover, it is worth noting that our method is extremely useful for enhancing the robustness of the multiple watermark embedding methods [4], [38], [44] (that are originally designed to resist geometric attacks) against estimation attacks.

Throughout this paper, we shall employ image watermarking to explain our method. In Section II, the watermark estimation attack that contains the collusion and copy attacks will be thoroughly explored. We show that conventional content-independent watermarking methods fail to resist WEA. Since "watermark estimation" is a necessary step in WEA, we will provide formal definitions of so-called "optimal watermark estimation" and "near-perfect cover data recovery" in Section III. We show that the proposed optimal watermark estimation/recovery method is an exquisite collusion attack that aims to actually eliminate the inserted watermark. From our analyses, we know that both accurate estimation of a watermark's sign and complete subtraction of a watermark's energy not only are indispensable for achieving effective watermark removal but also serve as clues to breaking WEA. In order to resist WEA, in Section IV content-dependent watermark (CDW) is proposed, which is composed of an informative watermark that carries information about an owner and a media hash that represents the essence of cover data. In addition, the validity of resistance to a collusion attack or copy attack using the CDW will be analyzed. Finally, experimental results and concluding remarks will be summarized in Sections V and VI, respectively.

\section{WATERMARK ESTIMATION ATTACKS}

Before addressing the issue of designing an anti-disclosure watermark, let's take a close look at the impact of watermark estimation attacks on the geometry-resilient methods proposed to hide multiple watermarks. Watermark attacks were roughly divided into four categories [43]: removal attacks, geometrical attacks, cryptographic attacks, and protocol attacks. Among them, removal attacks try to remove a hidden watermark by manipulating a watermarked image so that the quality of the attacked image is further destroyed. Let $\mathbf{X}, \mathbf{X}^{\mathbf{w}}$, and $\mathrm{X}^{\mathrm{a}}$ represent the original image, watermarked image, and attacked image, respectively. Therefore, for conventional removal attacks $\operatorname{PSNR}\left(\mathbf{X}, \mathbf{X}^{\mathbf{w}}\right) \geq \operatorname{PSNR}\left(\mathbf{X}, \mathbf{X}^{\mathbf{a}}\right)$ always holds. However, a more clever removal attack can achieve $\operatorname{PSNR}\left(\mathbf{X}, \mathbf{X}^{\mathbf{w}}\right) \leq \operatorname{PSNR}\left(\mathbf{X}, \mathbf{X}^{\mathbf{a}}\right)$; i.e., the attacked image $\mathbf{X}^{\mathbf{a}}$ is even closer (in terms of the mean square error) to the cover image $\mathbf{X}$ than the watermarked image $\mathbf{X}^{\mathbf{w}}$. The collusion attack is a typical example of an attack that follows the above scenario. Usually, a collusion attack is applied to video watermarking, as depicted in Fig. 1, by averaging a set of estimated watermarks to obtain the hidden watermark. As for image watermarking, some recent works have proposed embedding multiple redundant watermarks into local areas [4], [38], [44] so that global/local geometrical distortions can be resisted. Provided that we treat a local region in an image in a manner similar to the way in which we treat a video frame in a video, the same collusion attack can also be applied to region-based image watermarking. Once the hidden watermark is estimated, it can be subtracted from all the image blocks to yield the unwatermarked image. Under these 


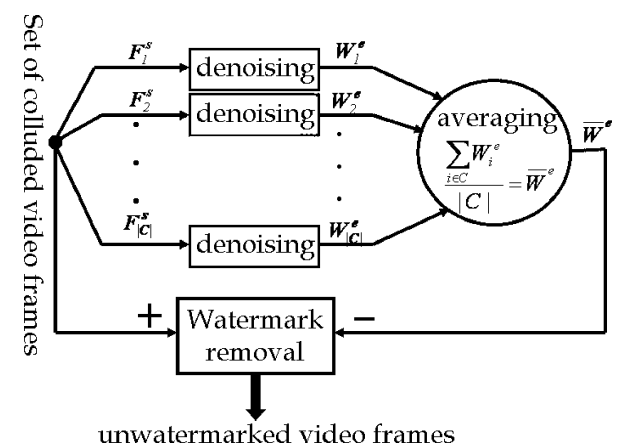

Fig. 1. Collusion attack on video frames: first, all video frames are embedded with the same watermark to form watermarked frames; second, denoising is used to estimate the hidden watermarks for visually dissimilar frames; third, the final watermark is determined by averaging those watermarks obtained in the second step and then subtracted from the watermarked frames to construct unwatermarked frames.

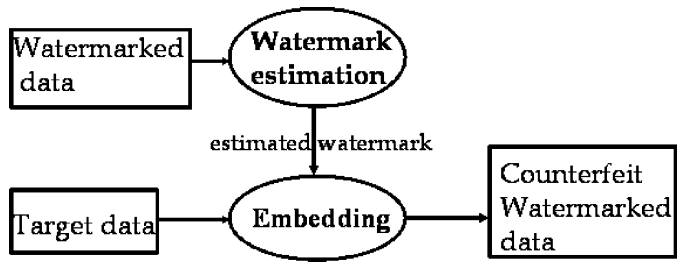

Fig. 2. Copy attack: watermark estimation can be accomplished by means of denoising without the need to know any secret key.

circumstances, the false negative problem will occur. It should be noted that the conventional denoising-based removal attack [42], which is only applied to a single image, is a special case of the collusion attack.

On the other hand, the estimated watermark can be inserted into unwatermarked media data to produce a counterfeit watermarked data. This is the so-called copy attack [17], which has been developed to create the false positive problem; i.e., the situation in which one can successfully detect a watermark from unwatermarked data. The copy attack, as depicted in Fig. 2, is a type of protocol attack. The copy attack is very simple to apply because it can be performed on only one piece of media data (e.g., an image).

Since watermark estimation is the first step in both attacks, these are called watermark estimation attack (WEA). In the next section, we shall compare the achievable performance between the denoising attack [42] (recall that it is a special case of the collusion attack) and the copy attack [17] to reveal the difficulty of resisting both of them simultaneously.

\section{A. Performance Comparison Between Denoising Attack and Copy Attack}

Let $\mathbf{Z}$ and $\mathbf{Z}^{\mathbf{w}}$ represent the faked original image and faked watermarked image, respectively. Recall that $\mathbf{X}^{\mathbf{w}}$ is generated from $\mathbf{X}$ through an embedding process. Here, $\mathbf{Z}^{\mathbf{w}}$ is generated from the combination of $\mathbf{Z}$ and a watermark extracted from $\mathbf{X}^{\mathbf{w}}$. In addition, let $\mathbf{W}$ be a watermark signal to be hidden in $\mathbf{X}$, and let $\mathbf{W}^{\mathbf{e}}$ be an estimated watermark obtained by denoising $\mathrm{X}^{\mathbf{w}}$. Without loss of generality, we use the bit-error rate (BER), which can be calculated in terms of Hamming distance, as a detection measurement between two bipolar/binary sequences.
By means of denoising-based watermark removal, $\mathbf{W}^{\mathbf{e}}$ will be subtracted from $\mathbf{X}^{\mathbf{w}}$ to produce an attacked image $\mathbf{X}^{\mathbf{a}}$, i.e.,

$$
\mathbf{X}^{\mathbf{a}}=\mathbf{X}^{\mathbf{w}}-\mathbf{W}^{\mathbf{e}}
$$

In the watermark detection process, a watermark, $\mathbf{W}^{\mathbf{a}}$, is extracted from $\mathbf{X}^{\mathbf{a}}$ and compared with $\mathbf{W}$. If denoising-based watermark removal is expected to succeed, then the BER between $\mathbf{W}$ and $\mathbf{W}^{\mathbf{a}}$ needs to be larger than a threshold.

As for the copy attack, the estimated watermark $\mathbf{W}^{\mathbf{e}}$ is added to the target image $\mathbf{Z}$ to form a counterfeit image $\mathbf{Z}^{\mathbf{w}}$, i.e.,

$$
\mathbf{Z}^{\mathbf{w}}=\mathbf{Z}+\mathbf{W}^{\mathbf{e}}
$$

In the watermark detection process, a watermark, $\mathbf{W}^{\mathbf{z}}$, is extracted from $\mathbf{Z}^{\mathbf{w}}$ and compared with $\mathbf{W}$. If the BER between $\mathbf{W}$ and $\mathbf{W}^{\mathbf{z}}$ is smaller than a threshold, then the copy attack is claimed to succeed.

Based on the above discussions, we know that a smaller threshold is advantageous to the resistance to copy attack while a larger threshold is advantageous to the resistance to denoising attack. This conflicting requirement clearly reveals the difficulty of resisting WEA simultaneously. Furthermore, we would like to emphasize that resistance to copy attack is relevant to reduction of false positive and resistance to denoising attack is relevant to reduction of false negative. If these two attacks are not properly dealt with, a watermarking system is easy to be defeated. On the other hand, if the denoised results (i.e., more than one estimated watermark) are collected and colluded to generate an estimation that is closer to its original, then the collusion attack will exhibit more powerful performance than the denoising attack, as evidenced in [35], [37].

To our knowledge, the collusion attack and the copy attack have not been simultaneously taken into consideration when investigating the robustness issue. In the next section, we shall provide the definitions of "optimal watermark estimation" and "nearperfect cover data recovery," based on which an approach that can combat the watermark-estimation attack will be addressed.

\section{OPtimal WATERMARK ESTIMATION FOR MULTIPLE WATERMARK EMBEDDING}

\section{A. How Watermark Could be Efficiently Estimated}

In the discussion of this section, the watermark $\mathbf{W}$ can be a bipolar sequence, a Gaussian sequence, or other distributions, and can represent 1-bit information or multiple-bit information. Without loss of generality, let $\mathbf{W}$ represent the original watermark with its energy extended by means of either a constant factor or a human visual system to enhance robustness [6]. From an attacker's perspective, the energy of each watermark value must be accurately predicted so that the previously added watermark energy can be completely eliminated to create an unwatermarked image. If this goal could be achieved, it is said that watermark removal is effective without leaving sufficient residual watermark. Consequently, an estimated watermark's energy is closely related to the effectiveness of the watermark removal attack. To better explain our point, several motivating scenarios are shown in Fig. 3, which illustrates the energy variations of (a) an original watermark; (b),(d) an estimated watermark 


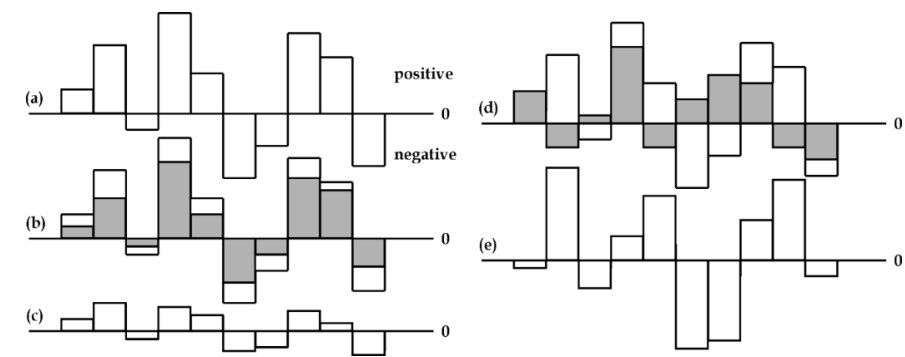

Fig. 3. Watermark estimation/removal illustrated with energy variation. (a) Original embedded watermark with each white bar indicating the energy (determined using perceptual masking) of each watermark value. (b) Gray bars show the energies of an estimated watermark with all the signs being the same as the originals (a). (c) The residual watermark obtained after removing the estimated watermark (b). (d) The energies of an estimated watermark with most the signs being opposite to those in (a). (e) The residual watermark derived from (d). In the above examples, sufficiently large correlations between (a) and (c), and between (a) and (e) exist, indicating the presence of a watermark.

(illustrated in grayscale); and (c),(e) a residual watermark generated by subtracting the estimated watermark from the original watermark. We can observe from Fig. 3(a)-(c) that even though the watermark's sign bits are fully obtained [Fig. 3(b)], the residual watermark signal [Fig. 3(c)] still suffices to reveal the hidden watermark due to the fact that the original watermark's energies cannot be completely discarded. Furthermore, if the sign of an estimated watermark value is different from its original one, then any additional energy subtraction will not be helpful in improving removal efficiency. On the contrary, watermark removal in terms of energy subtraction operated in the opposite (wrong) polarity will undesirably damage the media data's fidelity. Actually, this corresponds to adding a watermark with higher energy into cover data without satisfying the masking constraint, as shown in Fig. 3(d). After Fig. 3(d) is subtracted from Fig. 3(a), the resultant residual watermark is illustrated in Fig. 3(e). By comparing Fig. 3(a) and (e), it is highly possible to reveal the existence of a watermark.

In the watermark detection process, two strategies are usually used to indicate the existence of a watermark. The first strategy, called "watermark extraction," is to extract the signal from the watermarked or possibly attacked data as the form that it was previously embedded. The extracted signal can be correlated with the hidden one to show the presence/absence of a watermark, i.e., representing 1-bit information. On the other hand, the extracted signal can also be further decoded (if the signal is error correction encoded before embedding) to show the multi-bit information, whose correctness is measured in terms of BER. The second strategy, called "watermark detection," just detects the presence/absence of a watermark without explicitly representing it as an original form. In this regard, an earlier but nonoptimal correlator was proposed in [1]. In order to provide optimum solutions by properly modeling the host signal and the embedded signal, the so-called optimal watermark detectors have been proposed for additive watermark detection [11], [27] and multiplicative watermark detection [2], [5]. However, they still need to incorporate a synchronization mechanism to resist the geometric attacks. By taking the above two watermark detection strategies into consideration, we will now investigate how they are affected by the scenario of watermark estimation and removal, as depicted in Fig. 3.
As described in the beginning of Section II, video watermarking and region-based image watermarking are apt to suffer from the collusion attack. This is considered from the attacker's viewpoint. However, if we consider the same scenario from the owner's viewpoint, we shall attain a totally different result, i.e., we can gain an even accurate watermark estimation to enhance robustness. In this paper, we propose to use the "collusion estimation" to reliably estimate the hidden watermark. As a result, we will emphasize two essential points on the paradigm of geometric-resilient multiple watermark embedding approaches: 1) how to resist the collusion attack under reasonable false positive and 2) how to make use of collusion estimation to enhance robustness without relying on complex optimal detectors.

A representative image watermarking method with strong resistance to geometric distortions was proposed in [44], which embedded multiple watermarks, so that the hidden watermark pattern can be exploited to recover geometric distortions. However, if the watermarked image is modified by means of collusion such that the extracted watermark largely lose its original information (e.g., negative correlation, or small positive correlation with the hidden watermark), the geometric parameters cannot be accurately recovered. It should be noted that the same scenario works for other similar watermarking approaches [4], [38]. On the other hand, the existing optimal detectors [2], [5] [11], [27] that employ "watermark detection" strategy are naturally suitable for detecting watermarks from nongeometrically distorted images. If they are expected to deal with geometric distortions, the so-called synchronization problem needs to be dealt with. When the locally multiple watermarks are to be embedded [4], [38], [44], the image has to be divided into many regular/irregular regions. Under this circumstance, it is unclear whether those optimal watermark detectors are still feasible to model the region of small size accurately. Most importantly, the accuracy of watermark detection cannot be further improved by means of collusion estimation [22], [31] since the watermark detection strategy just provides the information about the presence/absence of a watermark.

The above observations motivate us to formulate the definitions of "optimal watermark estimation" and "near-perfect cover data recovery" based on Fig. 3 that are fundamental for introducing the hash-based content-dependent watermark to combat estimation attacks. In this study, optimal watermark estimation means that the hidden watermark can be completely removed. Therefore, if $\mathbf{W}^{\mathbf{e}}$ is estimated to be exactly equal to $\mathbf{W}$, then we immediately obtain $\operatorname{BER}\left(\operatorname{sgn}(\mathbf{W}), \operatorname{sgn}\left(\mathbf{W}^{\mathbf{e}}\right)\right)=0$. This leads to Definition 1, described below.

Definition 1 (Necessary Condition for Optimal Watermark Estimation): Given an originally embedded watermark signal $\mathbf{W}$ and its approximate version $\mathbf{W}^{\mathbf{e}}$ estimated from $\mathbf{X}^{\mathbf{w}}$, the necessary condition for the optimal estimation of $\mathbf{W}$ as $\mathbf{W}^{e}$ is defined as ${ }^{2}$

$$
\operatorname{BER}\left(\operatorname{sgn}(\mathbf{W}), \operatorname{sgn}\left(\mathbf{W}^{\mathbf{e}}\right)\right) \leq \tau
$$

in the sense that the BER calculated between $\operatorname{sgn}(\mathbf{W})$ and $\operatorname{sgn}\left(\mathbf{W}^{\mathbf{e}}\right)$ in terms of Hamming distance can be as small as

\footnotetext{
${ }^{2}$ With respect to (3), the capability of a collusion attack with regard to the optimal estimation of a watermark's sign is further justified in the Appendix.
} 
possible, where $\operatorname{sgn}(\mathbf{W})$ stands for the sign vector of $\mathbf{W}$. In (3), $\tau$ is a sufficiently small value and the sign function, $\operatorname{sgn}(\cdot)$, for an element is defined as

$$
\operatorname{sgn}(t)= \begin{cases}+1, & \text { if } t \geq 0 \\ -1, & \text { if } t<0\end{cases}
$$

In Definition 1, $\tau$ is basically watermarking algorithm- and application-dependent. To assist our later analysis, we use $\Theta$ to denote the set of indexes satisfying $\operatorname{sgn}\left(W^{e}(l)\right)=\operatorname{sgn}(W(l))$ in (3). Definition 1 is naturally derived from Fig. 3 in that the "polarity" 3 of each watermark value is particularly crucial. This is the first step, where the existence of a watermark may be efficiently eliminated if most sign bits of the watermark can be obtained by an attacker. Beyond this step, however, to avoid leaving a residual watermark [as illustrated in Fig. 3(c)] that can reveal the hidden watermark, accurate estimation of the energy of $\mathbf{W}^{\mathbf{e}}$ is absolutely indispensable. Thus, a sufficient and necessary condition for optimal watermark estimation is defined as follows.

Definition 2 (Sufficient and Necessary Condition for Optimal Watermark Estimation): Assuming that most of the signs of watermark values can be obtained as given in Definition 1, then complete watermark removal can be nearly achieved if the watermark energy to be subtracted is also larger than or equal to the added energy, i.e., $\operatorname{mag}\left(W^{e}(l)\right) \geq \operatorname{mag}(W(l))$, where $\operatorname{mag}(t)$ denotes the magnitude $|t|$ of $t$ and $l \in \Theta$. Therefore, it is said that $\mathbf{W}^{\mathbf{e}}$ is an optimal estimation of $\mathbf{W}$ if and only if

$$
\begin{aligned}
\operatorname{BER}\left(\operatorname{sgn}(\mathbf{W}), \operatorname{sgn}\left(\mathbf{W}^{\mathbf{e}}\right)\right) & \leq \tau \text { and } \\
\operatorname{mag}\left(W^{e}(l)\right) & \geq \operatorname{mag}(W(l)) \quad \forall l \in \Theta .
\end{aligned}
$$

In Definition 2, if (4) is satisfied, then there is no doubt that $\mathbf{W}^{\mathbf{e}}$ is an optimal estimation of $\mathbf{W}$. Thus, the sufficient condition holds. As for the part of necessary condition, since the goal of optimal watermark estimation discussed here originates from the viewpoint of complete removal of the hidden watermarks instead of from some similarity metrics (such as correlations), if the estimated watermark $\mathbf{W}^{\mathbf{e}}$ is equal to the original watermark $\mathbf{W}$, then (4) must be satisfied. That is why (4) is also the necessary condition. As described in Fig. 3, Fig. 3(a)-(c) illustrate that in some correlation metrics the original watermark in Fig. 3(a) and the estimated watermark in Fig. 3(b) (in gray bars) are highly correlated but the residual watermark shown in Fig. 3(c) may still reveal the hidden information. Furthermore, we would like to stress that the above definitions are irrelevant to the (optimal or nonoptimal) characteristic of a watermark detector. On the contrary, the key is whether or not the previously added watermark's sign bits and energies could be mostly removed.

It becomes evident from (4) that after removing $\mathbf{W}^{\mathbf{e}}$ only few watermark bits can survive. In order to make a collusion attack useless, it is necessary to force the attackers to get $\operatorname{BER}\left(\operatorname{sgn}(\mathbf{W}), \operatorname{sgn}\left(\mathbf{W}^{\mathbf{e}}\right)\right) \approx 0.5$ for each embedding unit (under this situation, $\mathbf{W}$ is embedded into each embedding unit [4], [38], [44]), which is equivalent to a random guess; i.e., half of the watermark's sign bits are correctly guessed and the

\footnotetext{
${ }^{3}$ The importance of the polarities of watermark values has been previously emphasized in [20], where two complementary embedded watermarks were modulated in different ways to resist different sets of attacks and the prior knowledge of a watermark detector was exploited to design a watermarking scheme that could resist both denoising and copy attacks.
}

other half are wrongly guessed. Therefore, we will propose in Section IV-A a media hash extraction method for constructing a so-called content-dependent watermark that attempts to lower the confidence of collusion [as derived in (23)]. Based on the derived optimal watermark estimation, near-perfect cover data recovery is defined as follows.

Definition 3 (Perfect Cover Data Recovery): Under the prerequisite that Definition 2 is satisfied and $\tau \approx 0$, it can be said that $\mathbf{X}^{\mathbf{r}}$ is a perfect recovery of $\mathbf{X}$ if the PSNR measure between them approximates infinity or the mean squared error (MSE)-based distortion measure between them approximates zero. That is

$$
\operatorname{PSNR}\left(\mathbf{X}, \mathbf{X}^{\mathbf{r}}\right) \approx \infty \quad \text { or } \quad D\left(\mathbf{X}, \mathbf{X}^{\mathbf{r}}\right) \approx 0
$$

where $\mathbf{X}^{\mathbf{r}}=\mathbf{X}^{\mathbf{w}}-\operatorname{sgn}\left(\mathbf{W}^{\mathbf{e}}\right) \operatorname{mag}\left(\mathbf{W}^{\mathbf{e}}\right), \mathbf{X}^{\mathbf{w}}=\mathbf{X}+$ $\operatorname{sgn}(\mathbf{W}) \operatorname{mag}(\mathbf{W})$, and $\operatorname{sgn}(\mathbf{v})$ and $\operatorname{mag}(\mathbf{v})$ are two vectors representing the sign and magnitude of the elements in a vector $\mathbf{v}$, respectively. $D(\cdot, \cdot)$ stands for the distortion measure based on MSE.

It is noted that ideally (5) is satisfied only if $\operatorname{mag}\left(\mathbf{W}^{\mathbf{e}}\right) \approx$ $\operatorname{mag}(\mathbf{W})$; otherwise, even if the watermark values have been completely removed based on, for example, $\operatorname{mag}\left(\mathbf{W}^{\mathbf{e}}\right) \gg$ $\operatorname{mag}(\mathbf{W})$, the quality of the attacked/recovered image would be undesirably degraded. Typically, evaluation of $\operatorname{mag}\left(\mathbf{W}^{\mathbf{e}}\right)$ can be achieved by means of either averaging [37] or perceptual remodulation [42]. It should be noted that if the residual watermark [Fig. 3(c)] becomes empty or negatively correlated with the hidden watermark [Fig. 3(a)], then even optimal watermark detector is unable to detect the hidden watermark (otherwise; false positive occurs). Definition 3 has specified how a cover data could be recovered in a near-perfect manner. In the next section, a near-perfect cover data recovery algorithm will be described.

\section{B. A Near-Perfect Cover Data Recovery Algorithm}

"Near-perfect" here means that the hidden watermarks can be mostly removed with a high probability (e.g., $\tau$ of (4) is sufficiently small but not zero) so that the recovered data is more similar to the cover data than the watermarked data in terms of PSNR. Under this circumstance, it is not necessary to worry about the detection ability of optimal watermark detector; otherwise, they will run the risk of raising the false positive problem. Here, we shall take the block-based multiple self-reference watermarking method [44] as an example to explain the performance of our algorithm in removing the hidden watermarks. However, it should be noted that our algorithm can be extended to other methods [4], [38] that adopt the similar concept of multiple redundant watermark embedding.

In the following, the method [44] where the watermark embedded in each image block is a bipolar sequence $\mathbf{w}$ is briefly described. This watermark $\mathbf{w}$ is flipped and copied in each direction to produce a symmetric signal, which is repeated over the entire image. In the embedding process, both the expanded watermark signal and the cover image are first decomposed using wavelet transform. Then, the watermark signals are embedded into the cover image in the wavelet domain through linear additive modulation together with a perceptual masking model called "noise visibility function (NVF)" [41]. The NVF is basically a wavelet-based content-adaptive visual model so that the 
degree for each wavelet coefficient that can be modified without raising perceptual difference can be defined. Let $\mathrm{NVF}_{k, l}(m, n)$ denote the masking threshold for the wavelet coefficient at the position $(m, n)$ of subband $k, l$ (where $k$ denotes scale and $l$ denotes orientation), and let $x_{k, l}(m, n)$ and $y_{k, l}(m, n)$ denote the cover and watermarked image wavelet coefficients, respectively. They are related as

$$
\begin{array}{r}
y_{k, l}(m, n)=x_{k, l}(m, n)+\left(1-\mathrm{NVF}_{k, l}(m, n)\right) \cdot w_{k, l}(m, n) \cdot S_{k, l}^{e} \\
+\operatorname{NVF}_{k, l}(m, n) \cdot w_{k, l}(m, n) \cdot S_{k, l}^{f}
\end{array}
$$

where $w_{k, l}(m, n)$ 's denote the watermark wavelet coefficients of $\mathbf{w}, S_{k, l}^{e}$, and $S_{k, l}^{f}$ denote the embedding strength [41] for nonflat and flat regions, respectively.

Now, the proposed near-perfect cover data recovery algorithm based on the collusion estimation of watermark's signs and NVF-based estimation of watermark's magnitudes is described, respectively, as follows.

1) Collusion Estimation of Watermark Sign: Let $\mathbf{W}^{\mathrm{e}}$ be the watermark finally estimated by means of collusion. In our implementation, for each embedding unit (e.g., image block or mesh) with index $q$, we adopt Wiener filtering for denoising purpose to get an estimation $\mathbf{W}_{\mathbf{q}}^{\mathbf{e}}$ of the hidden watermark $\mathbf{W}$. Recalled that we have defined in Section III-A that $\mathbf{W}$ represents the hidden watermark $\mathbf{w}$ (a bipolar sequence) with its energy extended by means of either a constant factor or a human visual system. Next, two vectors, $\operatorname{sgn}\left(\mathbf{W}_{\mathbf{q}}^{\mathbf{e}}\right)$ and $\operatorname{mag}\left(\mathbf{W}_{\mathbf{q}}^{\mathbf{e}}\right)$, regarding the sign and magnitude of $\mathbf{W}_{\mathbf{q}}^{\mathbf{e}}$ are calculated. Let $N$ denote the length of a watermark and let $Q(1 \leq q \leq Q)$ be the number of image units (or watermarks) in an image. In practice, each element, $\operatorname{sgn}\left(W^{e}(t)\right)$, of $\operatorname{sgn}\left(\mathbf{W}^{\mathbf{e}}\right)$ is finally determined to be either +1 or -1 depending on which one appears more than half. That is

$$
\operatorname{sgn}\left(W^{e}(t)\right)= \begin{cases}+1, & \text { if } \sum_{q=1}^{Q} \operatorname{sgn}\left(W_{q}^{e}(t)\right)>0 \\ -1, & \text { otherwise }\end{cases}
$$

where $1 \leq t \leq N$. Once the sign vector of the watermark is determined, the magnitude vector of the watermark is then estimated as follows.

2) Estimation of Watermark Magnitude via Visual Model for Complete Removal: As pointed out in Fig. 3 and Definition 3, accurate estimation of watermark's magnitudes is crucial to completely remove the hidden watermarks. In fact, we would rather remove more watermark energy than it should be so that the watermark energy can be more guaranteed to be eliminated. Therefore, the wavelet coefficient for the recovered image $\mathbf{X}^{\mathbf{r}}$ based on Definition 3 can be derived as

$$
\begin{aligned}
& z_{k, l}(m, n)=y_{k, l}(m, n)-\left[\left(1-\mathrm{NVF}_{k, l}^{w}(m, n)\right) \cdot w_{k, l}^{e}(m, n)\right. \\
& \left.\cdot S_{k, l}^{e}+\mathrm{NVF}_{k, l}^{w}(m, n) \cdot w_{k, l}^{e}(m, n) \cdot S_{k, l}^{f}\right]\left(1+\epsilon_{k, l}(m, n)\right)
\end{aligned}
$$

where $w_{k, l}^{e}(m, n)$ 's denote the estimated watermark wavelet coefficients, $\operatorname{NVF}_{k, l}^{w}(m, n)$ 's denote the masking thresholds estimated from a watermarked image, and $\epsilon_{k, l}(m, n)$ 's are mainly used to more guarantee that the hidden watermark can be completely removed. More specifically, if all the parameters $w_{k, l}^{e}(m, n)$ 's, $\mathrm{NVF}_{k, l}^{w}(m, n)$ 's, $S_{k, l}^{e}$ 's, and $S_{k, l}^{f}$ 's can be accurately estimated, then it suffices to set $\epsilon_{k, l}(m, n)=0$ to completely remove the hidden watermark. However, to take estimation errors into account $\epsilon_{k, l}(m, n)>0$ is set to remove more embedded watermark energy than expected. Our experimental results will show the tradeoff between the degree of watermark removal and fidelity degradation. By substituting (6) into (8) and assuming that the recovered image is equal to the cover image; i.e., $z_{k, l}(m, n)=x_{k, l}(m, n)$ for all $k, l, i$, and $j$, the desired parameters can be derived as in (9), shown at the bottom of the page.

To make (9) solvable, we further assume that the original and estimated watermark wavelet coefficients satisfy $w_{k, l}(m, n)=$ $w_{k, l}^{e}(m, n)$ for all $k, l, m$, and $n$. Since the estimation of the watermark's sign is rather accurate in our method, which reasonably approximates the assumption. In this case, $\epsilon_{k, l}(m, n)$ can be ideally derived as

$$
\epsilon_{k, l}(m, n)=\frac{\operatorname{NVF}_{k, l}(m, n)-\mathrm{NVF}_{k, l}^{w}(m, n)}{\mathrm{NVF}_{k, l}^{w}(m, n)-\frac{S_{k, l}^{e}}{S_{k, l}^{e}-S_{k, l}^{f}}} .
$$

In (10), $\mathrm{NVF}_{k, l}(m, n)$ 's are still unknown since no cover data is available in a blind detection scenario to obtain its NVF. However, they can be approximately estimated if $\mathbf{X}^{\mathbf{w}}-\operatorname{sgn}\left(\mathbf{W}^{\mathbf{e}}\right) \operatorname{mag}\left(\mathbf{W}^{\mathbf{e}}\right)$, as described in Definition 3, is used to obtain an approximate cover image. This is reasonable because similar images will have similar noise visibility function. It should be noted that when $\epsilon_{k, l}(m, n)$ 's are equal to zero, this algorithm degenerates to watermark remodulation [42].

In this section, we have derived how the watermark sign and watermark magnitude can be separately estimated to achieve complete watermark removal. In order to evaluate the performance of "near-perfect cover data recovery," it is best to compare the recovered image with the cover image to check how many watermark bits still survive in the recovered image. On the other hand, in order to enhance the robustness of multiple redundant watermark embedding approaches against watermark-estimation attacks, we shall propose in Section IV a new content-dependent watermark, which is a hybrid of a robust media hash and a watermark.

\section{Remarks}

In [34], Su and Girod proposed energy-efficient watermarking based on power-spectrum condition (PSC), which theoretically confirms that the watermark's power spectrum should be directly proportional to the cover data's power spectrum, while this result

$$
\epsilon_{k, l}(m, n)=\frac{\left(1-\operatorname{NVF}_{k, l}(m, n)\right) \cdot w_{k, l}(m, n) \cdot S_{k, l}^{e}+\operatorname{NVF}_{k, l}(m, n) \cdot w_{k, l}(m, n) \cdot S_{k, l}^{f}}{\left(1-\operatorname{NVF}_{k, l}^{w}(m, n)\right) \cdot w_{k, l}^{e}(m, n) \cdot S_{k, l}^{e}+\operatorname{NVF}_{k, l}^{w}(m, n) \cdot w_{k, l}^{e}(m, n) \cdot S_{k, l}^{f}}-1
$$


matches the heuristic arguments in previous visual model-based watermarking approaches (please refer [6] and the references therein). The authors concluded that a PSC-compliant watermark cannot be efficiently estimated by means of Wiener filtering.

Although our method exploits Wiener filtering to get an estimation of a hidden watermark, there, in fact, exists major differences between our method and [34]. First, our objective investigates the collusion estimation to reveal the weakness of multiple watermark embedding methods, while [34] did not consider the collusion attack. Second, an important assumption made in [34] is the use of a fixed detector, while our method can be adaptive to any detector. Third, when PSC-based watermarking and Wiener attack are considered, Su and Girod's method achieves either $D\left(\mathbf{X}, \mathbf{X}^{\mathbf{r}}\right) \approx D\left(\mathbf{X}, \mathbf{X}^{\mathbf{w}}\right)$ for removal (Wiener denoising) attack or $D\left(\mathbf{X}, \mathbf{X}^{\mathbf{r}}\right) \gg D\left(\mathbf{X}, \mathbf{X}^{\mathbf{w}}\right)$ for anti-correlation attack. However, our method is able to achieve $D\left(\mathbf{X}, \mathbf{X}^{\mathbf{r}}\right) \ll D\left(\mathbf{X}, \mathbf{X}^{\mathbf{w}}\right)$. Basically, the aforementioned differences are naturally resulted from the fact that we consider collusion estimation for multiple watermark embedding approaches, and the goals of our method and the method in [34] are, thus, not the same.

\section{CONTENT-DePEndent Watermark}

In this section, a robust media hashing mechanism is first described. Then, the properties of the content-dependent watermark is discussed. Finally, its resistance to WEA is analyzed based on block-based image watermarking.

\section{A. Media Hash}

From the analyses of the watermark-estimation attack described in Section II, we have found that the success of the WEA mainly depends on the fact that the hidden watermark totally behaves like a noise and can be easily and reliably obtained by means of a denoising process [14], [17], [44]. In order to disguise this prior knowledge and hide it from attackers, a watermark must be designed to carry information relevant to the cover image itself. It should be noted that the so-called cover image related information here means the unique feature of an image. Meanwhile, the content-dependent information must be secured $^{4}$ by means of a secret key and be robust to digital processing [21] in order not to affect watermark detection. To this end, we shall introduce the idea of the media hash as a kind of content-dependent information used to create the so-called content-dependent watermark (CDW).

The media hash [10], [24], also known as the "digital signature" [19], [21], [36] or "media fingerprint" [28], [29], has been used in many applications, including content authentication, copy detection, and media recognition. In this paper, the proposed image hash extraction algorithm ${ }^{5}$ that can work with

\footnotetext{
${ }^{4}$ This is because either an owner or an attacker can freely derive contentdependent information. Hence, a secret key is required for shuffling to enhance security.

${ }^{5}$ Among the existing media hashing methods, Fridrich proposed a robust/visual hash function for digital watermarking [10]. The hash digests of digital images were created by projections of DCT coefficients to key-dependent random patterns based on the observation that large changes to low-frequency DCT coefficients will significantly change the quality of an image. However, the pattern size is an important factor that will affect the robustness of the hash sequence. Since we need to perform multiple watermark embedded, the embedding unit should be small. In addition, based on our earlier studies [22], it is found that the block hash generated based on pairwise transformed coefficient comparison in our paper works well for resistance to the collusion and copy attacks.
}

the block-based image watermarking method (e.g., [44]) and adapt to video watermarking standards is introduced in the $8 \times 8$ block-DCT domain. However, we would like to particularly emphasize that for different watermarking algorithms (e.g., [4], [38]), the proposed media hash extraction method can be adjusted accordingly.

For a pair of $8 \times 8$ blocks, a piece of representative and robust information is created. It is defined as the magnitude relationship between two AC coefficients at blocks $u$ and $v$

$$
r(k)= \begin{cases}+1, & \text { if }\left|f_{u}\left(p_{1}\right)\right|-\left|f_{v}\left(p_{2}\right)\right| \geq 0\left(p_{1} \neq p_{2}\right) \\ -1, & \text { otherwise }\end{cases}
$$

where $r(k)$ is an element of a feature sequence $\mathbf{r}$ and $f_{u}\left(p_{1}\right)$ denotes an AC coefficient at position $p_{1}\left(0 \leq p_{1} \leq 63\right)$ in block $u$. The DC coefficients will not be selected because they are not sufficient to represent the unique features of image blocks. In addition, the selected AC coefficients should be at lower frequencies because high-frequency coefficients are vulnerable to attacks. In this paper, $p_{1}$ and $p_{2}$ are selected as the first two AC coefficients (i.e., $p_{1}=1, p_{2}=2$ ) from 64 available frequency subbands. As a result, two feature values are obtained from (11) by interchanging $p_{1}$ and $p_{2}$ for each pair of $8 \times 8$ blocks. It should be noted that although more than two DCT coefficients in a pair of $8 \times 8$ blocks can also be considered, this is related to how many hash bits are needed and what robustness the hash sequence can possess. This feature value $r(\cdot)$ is verified to be robust because this magnitude relationship between $f_{u}\left(p_{1}\right)$ and $f_{v}\left(p_{2}\right)$ can be mostly preserved under incidental modifications (e.g., compressions, filtering, and denoising). Basically, the proposed image hashing possesses the principle that is similar to our digital signature scheme [21], which is, however, not really robust against geometric distortions. To deal with this challenging issue, we have proposed a mesh-based content-dependent image hashing scheme [24]. Since the robustness of the media hash is beyond the scope of this paper, readers should refer to [24] for more results.

Let $M \times M$ be the block size of a "local region" where a watermark is embedded. Assume that $M$ is chosen as a multiple of 8 . Hence, the number of $8 \times 8$ blocks is $(M / 8)^{2}$, from which the total number of pairs of blocks will be

$$
\mathcal{P}\left(\begin{array}{c}
(M / 8)^{2} \\
2
\end{array}\right)
$$

where $\mathcal{P}$ denotes the permutation function. As a result, the length of the feature sequence $\mathbf{r}$ will be

$$
2 \mathcal{P}\left(\begin{array}{c}
(M / 8)^{2} \\
2
\end{array}\right) \text {. }
$$

Since a hash sequence and a watermark signal have to be combined to construct a content-dependent watermark, both of them must have the same length. As a result, the length of the feature sequence $\mathbf{r}$ is modified as follows such that its length is equal to the watermark's length. If

$$
2 \mathcal{P}\left(\begin{array}{c}
(M / 8)^{2} \\
2
\end{array}\right)
$$

is larger than watermark's length, then the extra elements at the tail of $\mathbf{r}$ are deleted; otherwise, $\mathbf{r}$ is cyclically extended. We call 
the finally created sequence media hash $\mathbf{M H}$, which is a bipolar sequence. Next, media hash $\mathrm{MH}$ of an image block is mixed with the watermark, $\mathbf{W}$, to generate the content-dependent watermark $(\mathbf{C D W})$ as

$$
\mathbf{C D W}=S(\mathbf{W}, \mathbf{M H})
$$

where $S(\cdot, \cdot)$ is a mixing function, which is operated based on a secret key and is used to prevent attackers from forging the CDW. This bipolar sequence CDW is what we will embed into a cover image.

\section{B. Properties}

Let an image $\mathbf{X}$ be expressed as $\cup_{i \in \Omega} \mathbf{B}_{i}$, where all blocks $\mathbf{B}_{i}$ are concatenated to form $\mathbf{X}$ and $\Omega$ denotes the set of block indices. As far as the block-based image watermarking scheme [44] is concerned, each image block $\mathbf{B}_{i}$ will be embedded with a content-dependent watermark $\mathbf{C D W}_{i}$ to form a watermarked image $\mathbf{X}^{\mathbf{w}}$, i.e.,

$$
\mathbf{B}^{\mathbf{w}}{ }_{i}=\mathbf{B}_{i}+E\left(\mathbf{C D W}_{i}\right), \quad \mathbf{X}^{\mathbf{w}}=\cup_{i \in \Omega} \mathbf{B}^{\mathbf{w}}{ }_{i}
$$

where $E(\cdot)$ is a watermark embedding procedure, and $\mathbf{B}^{\mathbf{w}}{ }_{i}$ is a watermarked block and $\mathbf{C D W}_{i}$, similar to (12), is defined as the mixture of a fixed informative watermark $\mathbf{W}$ and a block-based hash $\mathbf{M H}_{\mathbf{B}_{i}}$, i.e.,

$$
\mathrm{CDW}_{i}=S\left(\mathbf{W}, \mathbf{M H}_{\mathbf{B}_{i}}\right) .
$$

As $\mathbf{M H}_{\mathbf{B}_{i}}$ can be easily obtained by either owners or attackers, the mixing function $S(\cdot, \cdot)$ will be designed as a procedure for shuffling the media hash $\mathbf{M H}_{\mathbf{B}_{i}}$, followed by shuffling of the watermark to enhance security. Specifically, it is expressed as

$$
S\left(\mathbf{W}, \mathbf{M H}_{\mathbf{B}_{i}}\right)(k)=W(k) P T\left(\mathbf{M H}_{\mathbf{B}_{i}}, K\right)(k)
$$

where $P T$ denotes a random permutation controlled using the secret key $K$. Here, a transposition cipher [9], [16] is used to shuffle the position of a hash sequence with the aim of retaining robustness against bit errors (without error propagation). A more secure cryptographic cipher, such as the block cipher (e.g., the well-known DES algorithm), is not adopted due to its fragility to a single bit error. The security regarding shuffling (15) will further discussed in Section IV-B3.

The proposed content-dependent watermark possesses the characteristics described in the following. They are useful for proving resistance to WEA.

Definition 4 (Perceptual Similarity): Given two image blocks $\mathbf{B}_{i}$ and $\mathbf{B}_{j}$, their perceptual similarity is expressed in terms of BER measured between their corresponding media hashes, $\mathbf{M H}_{\mathbf{B}_{i}}$ and $\mathbf{M H}_{\mathbf{B}_{j}}$, i.e., $\operatorname{BER}\left(\mathbf{M H}_{\mathbf{B}_{i}}, \mathbf{M H}_{\mathbf{B}_{j}}\right)$. Accordingly, the smaller the $\operatorname{BER}\left(\mathbf{M H}_{\mathbf{B}_{i}}, \mathbf{M H}_{\mathbf{B}_{j}}\right)$ is, the more similar $\mathbf{B}_{i}$ and $\mathbf{B}_{j}$ are.

In fact, Definition 4 is derived from media hashing techniques [24], [32] that have been employed for measuring "similarity" of media contents for copy detection and searching.

Proposition 1: Given two image blocks $\mathbf{B}_{i}$ and $\mathbf{B}_{j}$, their perceptual similarity measurement $\operatorname{BER}\left(\mathbf{B}_{i}, \mathbf{B}_{j}\right)$, and their respectively embedded content-dependent watermarks $\mathbf{C D W}_{i}$ and $\mathbf{C D W}_{j}$, the following property can be established: $\operatorname{BER}\left(\mathbf{C D W}_{i}, \mathbf{C D W}_{j}\right)$ also serves as a similarity indicator in representing similarity between their corresponding image block contents $\mathbf{B}_{i}$ and $\mathbf{B}_{j}$.

Proof: Substitution of (14) and (15) into $\operatorname{BER}\left(\mathbf{C D W}_{i}, \mathbf{C D W}_{j}\right)$ will lead to

$$
\begin{aligned}
\operatorname{BER} & \left(\mathbf{C D W}_{i}, \mathbf{C D W}_{j}\right) \\
& =\operatorname{BER}\left(S\left(\mathbf{W}, \mathbf{M H}_{\mathbf{B}_{i}}\right), S\left(\mathbf{W}, \mathbf{M H}_{\mathbf{B}_{j}}\right)\right) \\
& =\operatorname{BER}\left(\mathbf{W} P T\left(\mathbf{M H}_{\mathbf{B}_{i}}, K\right), \mathbf{W} P T\left(\mathbf{M H}_{\mathbf{B}_{j}}, K\right)\right) \\
& =\operatorname{BER}\left(P T\left(\mathbf{M H}_{\mathbf{B}_{i}}, K\right), P T\left(\mathbf{M H}_{\mathbf{B}_{j}}, K\right)\right) \\
& =\operatorname{BER}\left(\mathbf{M H}_{\mathbf{B}_{i}}, \mathbf{M H}_{\mathbf{B}_{j}}\right) .
\end{aligned}
$$

Definition 4 can be used to interpret the result of (16). It is straightforward to know that Proposition 1 implies that similar/dissimilar image blocks will be embedded with similar/dissimilar watermarks that are content-dependent. This result contrasts with the one pointed out in [37], but the novelty of our scheme is that the idea of the content-dependent watermark has been employed. In addition, Proposition 1 also implies that the media hash is feasible for randomizing the hidden watermark. In particular, for visually dissimilar blocks this effect is remarkable, i.e., $\operatorname{BER}\left(\mathbf{C D W}_{i}, \mathbf{C D W}_{j}\right) \approx 0.5$.

1) Security of Shuffling and Protocol Attack: In order to break the shuffling function $((15))$ that is used to construct the content-dependent watermark, the success relies on the probability of inversing the shuffling function [23]. This corresponds to Single Watermarked Image Counterfeit Original (SWICO) attack, a kind of protocol attacks, first presented by Craver $e$ t al. [7]. Under the scenario of SWICO, Alice is the rightful owner and Bob is the attacker who exploits Alice's watermarked image as the available information to create his fake original image and fake watermark under the constraint that the watermarked image is kept unchanged. According to the Kerckhoff's principle, the attacker Bob is assumed to fully know the watermarking algorithm adopted by Alice except for the secret key. Accordingly, how much available information the attacker Bob can have and what kind of information Bob cannot know in advance is specifically described as follows.

Available Information: 1) the watermarked image; 2) the watermarking algorithm; and 3) the media hashing algorithm.

Unavailable Information: 1) the secret key used to generate the watermark; 2) the secret key used for shuffling.

In order to break the proposed watermarking scheme by means of SWICO, the attacker Bob needs to show he can produce the content-dependent watermark from his watermark and media hash [7]. Therefore, his success relies on the probability of inversing the shuffling function. In this study, we consider the ciphertext-only attack and the known-plaintext attack.

Let the length of a content-dependent watermark be $N$. Intuitively, the time-complexity of inversing one shuffling for the ciphertext-only attack with brute force is $O(N$ !). Thus, the total time-complexity is $O(|\Omega| \cdot N$ !), where $|\Omega|$ denotes the number of watermarks to be embedded. It is not hard to find that it is computationally impossible to inverse the employed shuffling without bit errors. 
However, more sophisticated attacks are possible if we assume that the content-dependent watermark and its corresponding media hash can be perfectly extracted from the watermarked image. This refers to the known plaintext attack. When an attacker, respectively, compares a pair of content-dependent watermarks and its corresponding pair of media hashes, partial permutation results are revealed. More specifically, an obtained permutation is partitioned into two subsets, where one set stands for the part that two hashes (and the CDWs) are the same and the other set represents those that are different. Based on this step, a total number of possible permutations is greatly reduced. By executing the above steps several times, it is possible to achieve the recovery of shuffling. In the worst case for attackers, at most $N-1$ such pairs are needed to fully achieve inverse one shuffle under the condition that the extracted content-dependent watermarks and media hashes do not incur errors. This implies that for each comparison a one-to-one position mapping of shuffling is found. Thus, the time complexity of inversing all shuffles in the worse case for an image is $O(|\Omega| \cdot N)$. In the best case for attackers, $\left\lceil\log _{2}^{N}\right\rceil$ pair comparisons are sufficient to break one shuffle under the condition that such pairs can be found. Thus, the time complexity of inversing all shuffles in the best case for an image is $O\left(|\Omega| \cdot\left\lceil\log _{2}^{N}\right\rceil\right)$.

Overall, the main differences distinguishing our approach from others in dealing with SWICO are that the length of a hidden watermark is not increased and blind detection is achieved.

\section{Resistance to Collusion Attacks}

Assume that by means of a collusion attack, the averaging operation is performed on watermarked blocks $\mathbf{B}^{\mathbf{w}}{ }_{i}$ 's of a watermarked image $\mathbf{X}^{\mathbf{w}}$. Let $\mathbf{W}^{\mathbf{e}}{ }_{i}$ be an estimated watermark from $\mathbf{B}^{\mathbf{w}}{ }_{i}$ and let it be modeled as a partial hidden watermark plus a noise component, i.e.,

$$
\mathbf{W}_{i}^{\mathbf{e}}=\alpha_{i} \mathbf{C D W}_{i}+\mathbf{n}_{i}
$$

where $\mathbf{n}_{i}$ represents an image block-dependent noise and $\alpha_{i}$ denotes the proportion that the hidden watermark has been extracted. This scalar $\alpha_{i}$ plays a crucial but conflicting role in two ways: 1) on one hand, from an attacker's viewpoint, $\alpha_{i}$ should be adjusted in a pixel/coefficient-wise manner so that perceptual fidelity can be maintained [42]; 2) on the other hand, from an owner's viewpoint, a watermarking system should be able to allow large $\alpha_{i}$ in order that strong attacks can be tolerated. Let $\mathcal{C}(\subset \Omega)$ denote the set of blocks used for collusion. By means of collusion, the average 6 of all the estimated watermarks can be expressed as

$$
\overline{\mathbf{W}}^{\mathbf{e}}=\frac{1}{|\mathcal{C}|} \sum_{i \in \mathcal{C}} \mathbf{W}^{\mathbf{e}}{ }_{i}=\frac{1}{|\mathcal{C}|} \sum_{i \in \mathcal{C}}\left(\alpha_{i} \mathbf{C D W}_{i}+\mathbf{n}_{i}\right)
$$

The next definition specifies the resistance of CDW to the collusion attack. Here, BER is used as a detection measurement since the method [44] belongs to multi-bit watermarking.

Definition 5: In a collusion attack, an attacker first estimates $\overline{\mathbf{W}}^{\mathrm{e}}$ from a set, $\mathcal{C}$, of image blocks. Then, a counterfeit unwa-

\footnotetext{
${ }^{6}$ Recall that final estimation of the hidden watermark can also be conducted using perceptual masking in order to maintain the fidelity of a colluded image, as previously discussed in Definition 3.
}

termarked image $\mathbf{X}^{\mathbf{u}}$ is generated from a watermarked image $\mathbf{X}^{\mathbf{w}}=\cup_{i \in \Omega} \mathbf{B}^{\mathbf{w}}{ }_{i}$ as

$$
\mathbf{B}_{i}^{\mathbf{u}}=\mathbf{B}^{\mathbf{w}}{ }_{i}-\overline{\mathbf{W}}^{\mathbf{e}}, \quad \mathbf{X}^{\mathbf{u}}=\cup_{i \in \Omega} \mathbf{B}^{\mathbf{u}}{ }_{i}
$$

It is said that the collusion attack fails in an image block $\mathbf{B}^{\mathbf{u}}{ }_{k}$, $k \in \Omega$, as long as $\operatorname{BER}\left(\overline{\mathbf{W}}^{e}, \mathbf{C D W}_{k}\right)$ is larger than a threshold.

Based on Definition 5, the next proposition further explains the power of our CDWs in resisting the collusion attack.

Proposition 2: According to the size of the collusion set, $|\mathcal{C}|$, the power of the collusion attack becomes lower bounded by the denoising-based removal attack when CDWs are embedded. That is, $\left.\operatorname{BER}\left(\overline{\mathbf{W}}^{e}, \mathbf{C D W}_{k}\right)\right|_{|\mathcal{C}| \neq 1} \geq$ $\left.\operatorname{BER}\left(\overline{\mathbf{W}}^{e}, \mathbf{C D W}_{k}\right)\right|_{|\mathcal{C}|=1}$.

Proof: Making use of (18) and Proposition 1, we have the following derivation:

$$
\begin{aligned}
& \operatorname{BER}\left(\overline{\mathbf{W}}^{\mathbf{e}}, \mathbf{C D W}_{k}\right) \\
& =\operatorname{BER}\left(\frac{1}{|\mathcal{C}|} \sum_{i \in \mathcal{C}}\left(\alpha_{i} \mathbf{C D W}_{i}+\mathbf{n}_{i}\right), \mathbf{C D W}_{k}\right) \\
& =\operatorname{BER}\left(\frac{\sum_{i \in \mathcal{C}} \alpha_{i} \mathbf{M H}_{B_{i}} \mathbf{W}}{|\mathcal{C}|}+\hat{\mathbf{n}}, \mathbf{M H}_{B_{k}} \mathbf{W}\right) \\
& \approx \operatorname{BER}\left(\frac{\sum_{i \in \mathcal{C}} \mathbf{M H}_{B_{i}}}{|\mathcal{C}|}, \mathbf{M H}_{B_{k}}\right)
\end{aligned}
$$

where the common term, $\mathbf{W}$, and the scalars, $\alpha_{i}$ 's, do not function in the BER function. In addition, in order to make the derivation of (20) analytic the average noisy term $\hat{\mathbf{n}}$ is reasonably assumed as a zero vector and can be ignored in particular when $|\mathcal{C}|$ is large enough. Provided that $|\mathcal{C}|$ becomes infinite, i.e., $|\mathcal{C}|=|\Omega| \rightarrow \infty, \operatorname{BER}\left(\overline{\mathbf{W}}^{\mathbf{e}}, \mathbf{C D W}_{k}\right)_{|\mathcal{C}| \rightarrow \infty} \rightarrow 0.5$ is expected since $\sum_{i \in \mathcal{C}} \mathbf{M H}_{B_{i}}$ can be statistically independent of $\mathbf{M H}_{B_{k}}$. On the other hand, if $|\mathcal{C}|=1$ (we mean that the collusion attack is only applied to one block), then the collusion attack degenerates into a denoising-based removal attack. Under this circumstance, the success of the collusion attack depends on the accuracy of estimation. Ideally, if denoising-based estimation is perfect without being affect by noise (e.g., $\mathbf{n}_{i}$ ), then $\operatorname{BER}\left(\overline{\mathbf{W}}^{\mathbf{e}}, \mathbf{C D W}{ }_{k}\right)_{|\mathcal{C}|=1}$ can be perfectly as low as zero that indicate the success of denoising. When $|\mathcal{C}|$ is larger than 1 but not infinite, the term $\sum_{i \in \mathcal{C}} \mathbf{M H}_{B_{i}}$ in (20) can be regarded as a noisy version of $\mathbf{M H}_{B_{k}}$. In this case, the result of (20) will be gradually deviated from 0 . Based on the above discussions, $\left.\operatorname{BER}\left(\overline{\mathbf{W}}^{e}, \mathbf{C D W}_{k}\right)\right|_{|\mathcal{C}| \neq 1} \geq\left.\operatorname{BER}\left(\overline{\mathbf{W}}^{e}, \mathbf{C D W}_{k}\right)\right|_{|\mathcal{C}|=1}$ can be derived, ${ }^{7}$ which implies that the proposed content-dependent watermarking scheme is unfavorable to the collusion attack, which is, by definition, applied to more than one image block. That is, when CDWs are applied, the inherent performance of a collusion attack is lost. This proves that when CDWs are embedded the power of the collusion attack becomes lower bounded by the denoising-based removal attack.

\footnotetext{
${ }^{7}$ Note that $\overline{\mathbf{W}}^{e}$ here is estimated from a watermarked image by means of a collusion attack. However, in the experiments described later the BER is calculated between the original watermark and the watermark extracted from a collusion attacked image. In other words, the BER values obtained here and later in the experiments refer to two different situations, i.e., before and after collusion.
} 


\section{Resistance to Copy Attack}

Next, we will proceed to show why the presented content-dependent watermark can be immune to a copy attack. Let $\mathbf{M H}_{\mathbf{u}}$ and $\mathbf{M H}_{\mathbf{v}}$ denote the hash sequences generated from two different image blocks, $\mathbf{u}$ and $\mathbf{v}$, respectively. In addition, let $\mathrm{CDW}_{\mathbf{u}}$ denote the content-dependent watermark to be hidden in the cover image block $\mathbf{u}$. As stated previously, let the watermark estimated from $\mathbf{u}^{\mathbf{w}}$ be $\mathbf{W}^{\mathbf{u}}$, which will contain partial information from $\mathbf{C D W}_{\mathbf{u}}$. By directing the copy attack at the target block $\mathbf{v}$, we can get the counterfeit watermarked block $\mathbf{v}^{\mathbf{w}}$ as defined in (2). Later, in the detection process, the content-dependent watermark, $\mathbf{W}^{\mathbf{v}}$, estimated from block $\mathbf{v}^{\mathbf{w}}$ will be

$$
\mathbf{W}^{\mathbf{v}}=\left(\alpha \times \mathbf{C D W}_{\mathbf{u}}+\mathbf{n}\right)
$$

according to (17), where $\mathbf{n}$ indicates the noise sequence generated by denoising $\mathbf{v}^{\mathbf{w}}$. Given (14) and (21), Proposition 1, and Definition 4, the BER between $\mathbf{C D W}_{\mathbf{v}}$ and $\mathbf{W}^{\mathbf{v}}$ can be derived as follows based on blocks $\mathbf{u}$ and $\mathbf{v}$ that are dissimilar:

$$
\begin{aligned}
\operatorname{BER}\left(\mathbf{C D W}_{\mathbf{v}}, \mathbf{W}^{\mathbf{v}}\right) & =\operatorname{BER}\left(\mathbf{C D W}_{\mathbf{v}}, \alpha \times \mathbf{C D W}_{\mathbf{u}}+\mathbf{n}\right) \\
& =\operatorname{BER}\left(\mathbf{M H}_{\mathbf{v}} \mathbf{W}, \alpha \times \mathbf{M H}_{\mathbf{u}} \mathbf{W}+\mathbf{n}\right) \\
& =\operatorname{BER}\left(\mathbf{M H}_{\mathbf{v}} \mathbf{W}, \mathbf{M H}_{\mathbf{u}}(\alpha \mathbf{W} \pm|\mathbf{n}|)\right) \\
& \approx 0.5
\end{aligned}
$$

where $|\mathbf{n}|$ denotes the magnitude sequence of $\mathbf{n}$. The above derivation could be interpreted in two ways. First, if the noise $\mathbf{n}$ can be ignored, (22) is directly derived based on Definition 4. Second, if $\mathbf{n}$ cannot be ignored, it can be said from the third line of (22) that $\mathbf{W}$ shuffles $\mathbf{M H}_{\mathbf{v}}$ and $(\alpha \mathbf{W} \pm|\mathbf{n}|)$ shuffles $\mathbf{M H}_{\mathbf{u}}$. Because both $\mathbf{M H}_{\mathbf{u}}$ and $\mathbf{M H}_{\mathbf{v}}$ are uncorrelated based on Definition 4, it is straightforward to know that both shuffled $\mathbf{M H}_{\mathbf{u}}$ and $\mathbf{M H}_{\mathbf{v}}$ are uncorrelated, too.

\section{EXPERIMENTAL RESULTS}

In our experiments, ten varieties of grayscale cover images of size $512 \times 512$, as shown in Fig. 4, were used for watermarking. In this study, Voloshynovskiy et al.'s block-based image watermarking approach [44] was chosen as the benchmark, denoted as Method I, due to its strong robustness and computational simplicity. However, we would like to particularly emphasize that the proposed CDW is readily applied to other watermarking algorithms that implement the similar principle of embedding multiple redundant watermarks [4], [38]. Each watermark of length 64 was embedded into an image block of size $32 \times 32$ and the number of blocks was $|\Omega|=256$. The number of hash bits generated in an image block (Section IV-A) was set to be equal to the watermark length. The combination of our CDW and Voloshynovskiy et al.'s scheme was denoted as Method II. Both methods were implemented in MATLAB and the noise visibility function of [44] was used to set embedding strength. Since Method I is a multi-bit watermarking scheme, we will use BER to measure the detection performance. On the other hand, Lee's Wiener filter [18] was used to perform denoising-based blind watermark extraction. In this study, we did not set a fixed threshold for final decision of the presence/absence of a water-

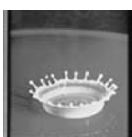

(X1)

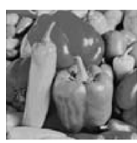

(X6)

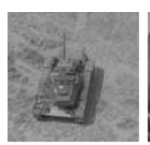

(X2)

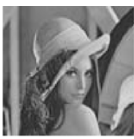

(X3)

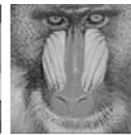

(X4)

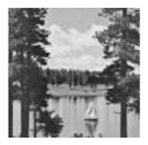

(X9)

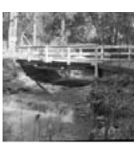

(X5)
Fig. 4. Cover images.

mark since this threshold is watermarking algorithm- or application-dependent. On the contrary, we show how the bit-error bit measured between the original and the extracted watermarks could be lowered if the proposed CDW is employed.

Four sets of experiments were conducted to demonstrate the advantage of deploying CDW. They are: 1) how watermarks could be removed using proposed optimal watermark estimation; 2) resistance of CDW to the collusion attack; 3) CDW versus false positive; and 4) resistance of CDW to the copy attack. We would like to demonstrate that 1) the proposed optimal watermark estimation/recover algorithm is effective to remove almost all watermark values so that the multiple redundant watermark embedding methods that were originally addressed to tolerate geometric distortions are successfully defeated; and 2) the proposed content-dependent watermark is able to resistance to both the collusion and copy attacks and is immune to false positive. Both the collusion and copy attacks were performed, as described in Section II.

\section{A. How Watermarks Could be Estimated/Removed Using Proposed Optimal Watermark Estimation}

We consider two scenarios in this section to demonstrate the performance of removing/estimating watermarks using our method. In the first scenario, we play the role of an owner who will try to estimate the hidden watermark's polarity using the proposed collusion estimation algorithm. The accuracy of this estimation may be disturbed when a noise adding attack is encountered. While in the second scenario, we play the role of an attacker who aims to remove the hidden watermarks using the proposed cover data recovery algorithm.

First, in order to verify how the hidden (content-independent) watermark's polarity could be retained by means of the proposed algorithm for collusion estimation of watermark sign (Section III-B1), the survived watermark of the obtained attacked (noise added) image was extracted using collusion estimation to check how many correct watermark bits still remain. Table I shows the BER values, which were obtained from comparing the original watermark and the extracted watermarks, and the PSNR values, which were calculated between the cover image and the attacked images.

As we can see from Table I that PSNRs have been decreased due to added noises and all BERs fall into the interval between $0.0 \% \sim 5.0 \%$, which means that the watermark's polarity is nearly retained. That is, the added noise is not able to disturb the collusion estimation of a watermark's polarity.

Second, in order to verify how the hidden (content-independent) watermark could be removed by means of the proposed 
TABLE I

VALidATION OF OUR COLLUSION Estimation SCHEME ON ATTACKED (NOISE ADDED) IMAGES. No CDW WAS EMBEDDED IN WATERMARKED IMAGES. BER IS COMPUTED BETWEen tHe ORIGINAL AND THE EXTRACTED WATERMARKS. PSNR IS COMPUTED BETWEen tHE COVER AND THE ATTACKED IMAGES

\begin{tabular}{l||l|l|l|l|l|l|l|l|l|l}
\hline watermarked image & $\mathbf{X}_{\mathbf{1}}^{\mathbf{w}}$ & $\mathbf{X}_{\mathbf{2}}^{\mathbf{w}}$ & $\mathbf{X}_{\mathbf{3}}^{\mathbf{w}}$ & $\mathbf{X}_{4}^{\mathbf{w}}$ & $\mathbf{X}_{5}^{\mathbf{w}}$ & $\mathbf{X}_{\mathbf{6}}^{\mathbf{w}}$ & $\mathbf{X}_{7}^{\mathbf{w}}$ & $\mathbf{X}_{\mathbf{8}}^{\mathbf{w}}$ & $\mathbf{X}_{\mathbf{9}}^{\mathbf{w}}$ & $\mathbf{X}_{\mathbf{1 0}}^{\mathbf{w}}$ \\
PSNR (dB) & 38.15 & 37.92 & 37.96 & 37.51 & 37.61 & 37.94 & 38.13 & 38.98 & 37.74 & 37.94 \\
\hline \hline Attacked (noise added) image & $\mathbf{X}_{\mathbf{1}}^{\mathbf{a}}$ & $\mathbf{X}_{\mathbf{2}}^{\mathbf{a}}$ & $\mathbf{X}_{\mathbf{3}}^{\mathbf{a}}$ & $\mathbf{X}_{4}^{\mathbf{a}}$ & $\mathbf{X}_{5}^{\mathbf{a}}$ & $\mathbf{X}_{\mathbf{6}}^{\mathbf{a}}$ & $\mathbf{X}_{7}^{\mathbf{a}}$ & $\mathbf{X}_{\mathbf{8}}^{\mathbf{a}}$ & $\mathbf{X}_{9}^{\mathbf{a}}$ & $\mathbf{X}_{10}^{\mathbf{a}}$ \\
PSNR (dB) & 29.56 & 29.34 & 29.41 & 29.29 & 29.31 & 29.59 & 29.37 & 29.50 & 29.36 & 29.37 \\
BER (\%) & 3.1 & 0.0 & 1.6 & 1.6 & 4.7 & 1.6 & 4.7 & 1.6 & 1.6 & 0.0 \\
\hline
\end{tabular}

TABLE II

VALIDATION OF OUR ESTIMATION AND RECOVERY SCHEME. NO CDW WAS EMBEDDED IN WATERMARKED IMAGES. BER IS COMPUTED BETWEEN THE ORIGINAL AND THE EXTRACTED WATERMARKS. PSNR IS COMPUTED BETWEEN THE COVER AND THE RECOVERED/WATERMARKED IMAGES

\begin{tabular}{|c|c|c|c|c|c|c|c|c|c|c|}
\hline $\begin{array}{l}\text { watermarked image } \\
\text { PSNR }(\mathrm{dB})\end{array}$ & $\begin{array}{l}\mathbf{X}_{1}^{\mathbf{w}} \\
38.15\end{array}$ & $\begin{array}{l}\mathbf{X}_{2}^{\mathbf{w}} \\
37.92\end{array}$ & $\begin{array}{l}\mathbf{X}_{3}^{\mathbf{w}} \\
37.96\end{array}$ & $\begin{array}{l}\mathbf{X}_{4}^{\mathbf{w}} \\
37.51\end{array}$ & $\begin{array}{l}\mathbf{X}_{5}^{\mathbf{w}} \\
37.61\end{array}$ & $\begin{array}{l}\mathbf{X}_{6}^{\mathbf{w}} \\
37.94\end{array}$ & $\begin{array}{l}\mathbf{X}_{7}^{\mathbf{w}} \\
38.13\end{array}$ & $\begin{array}{l}\mathbf{X}_{\mathbf{8}}^{\mathbf{w}} \\
38.98\end{array}$ & $\begin{array}{l}\mathbf{X}_{\mathbf{9}}^{\mathbf{w}} \\
37.74\end{array}$ & $\begin{array}{l}\mathbf{X}_{10}^{\mathbf{w}} \\
37.94\end{array}$ \\
\hline $\begin{array}{l}\text { Recovered image } \\
\text { (using } \epsilon_{k, l}(m, n)=0 \text { [42]) }\end{array}$ & $\mathrm{X}_{1}^{\mathrm{r}}$ & $\mathrm{X}_{2}^{\mathrm{r}}$ & $\mathrm{X}_{3}^{\mathrm{r}}$ & $\mathrm{X}_{4}^{\mathrm{r}}$ & $X_{5}^{r}$ & $\mathrm{X}_{6}^{\mathrm{r}}$ & $\mathrm{X}_{7}^{\mathrm{r}}$ & $\mathrm{X}_{8}^{\mathrm{r}}$ & $X_{9}^{r}$ & $\mathrm{X}_{10}^{\mathrm{r}}$ \\
\hline PSNR $(\mathrm{dB})$ & 45.46 & 53.25 & 53.16 & 43.10 & 56.20 & 48.18 & 52.79 & 45.05 & 54.83 & 53.25 \\
\hline BER (\%) & 63.4 & 50.7 & 63.3 & 51.7 & 55.6 & 64.2 & 74.0 & 76.2 & 65.8 & 60.6 \\
\hline $\begin{array}{l}\text { Recovered image } \\
\text { (using } \epsilon_{k, l}(m, n) \neq 0 \text { ) }\end{array}$ & $\mathrm{X}_{1}^{\mathrm{r}}$ & $\mathrm{X}_{2}^{\mathrm{r}}$ & $\mathrm{X}_{3}^{\mathrm{r}}$ & $\mathrm{X}_{4}^{\mathrm{r}}$ & $X_{5}^{r}$ & $\mathrm{X}_{6}^{\mathrm{r}}$ & $\mathrm{X}_{7}^{\mathrm{r}}$ & $\mathrm{X}_{8}^{\mathrm{r}}$ & $X_{9}^{r}$ & $\mathrm{X}_{10}^{\mathrm{r}}$ \\
\hline PSNR (dB) & 53.15 & 55.30 & 54.38 & 59.65 & 58.28 & 53.44 & 53.31 & 49.06 & 56.08 & 54.23 \\
\hline BER (\%) & 82.1 & 85.7 & 85.1 & 89.6 & 89.4 & 90.5 & 86.3 & 80.5 & 87.8 & 84.0 \\
\hline $\begin{array}{l}\text { Recovered image } \\
\text { (using } 2 \times \epsilon_{k, l}(m, n) \text { ) }\end{array}$ & $\mathrm{X}_{1}^{\mathrm{r}}$ & $\mathrm{X}_{2}^{\mathrm{r}}$ & $\mathrm{X}_{3}^{\mathrm{r}}$ & $\mathrm{X}_{4}^{\mathrm{r}}$ & $\mathrm{X}_{5}^{\mathrm{r}}$ & $\mathrm{X}_{6}^{\mathrm{r}}$ & $\mathrm{X}_{7}^{\mathrm{r}}$ & $\mathrm{X}_{8}^{\mathrm{r}}$ & $\mathrm{X}_{9}^{\mathrm{r}}$ & $\mathrm{X}_{10}^{\mathrm{r}}$ \\
\hline PSNR (dB) & 51.69 & 53.53 & 52.90 & 57.12 & 56.06 & 51.52 & 52.16 & 47.65 & 54.45 & 52.34 \\
\hline BER (\%) & 88.5 & 92.8 & 93.0 & 93.8 & 94.1 & 93.7 & 92.8 & 81.8 & 92.8 & 90.9 \\
\hline
\end{tabular}

cover data recovery algorithm (Section III-B), the survived watermark of the obtained recovered image was extracted using the cover image so that we can accurately check how many correct watermark bits still remain. Table II shows the BER values, which were obtained from comparing the original watermark and the extracted watermarks, and the PSNR values, which were calculated between the cover image and the recovered/watermarked image. As we can see from Table II that if $\epsilon_{k, l}(m, n)=0$ is used, this corresponds to perceptual remodulation [42]. It is observed that PSNRs have been increased and most BERs fall into the interval between $50 \% \sim 65 \%$, which means that a significant part of watermark values is not completely removed.

However, if $\epsilon_{k, l}(m, n) \neq 0$ is adopted, BERs can be increased averagely as high as 0.9 except for some very smoothing images, which implies that our estimation and recovery algorithms are able to remove almost all the watermark bits in a watermarked image. In addition, the obtained PSNRs are further improved than those obtained using [42] such that the recovered image can be more similar to its cover version. Since $\epsilon_{k, l}(m, n)$ 's are approximated derived in (10), if $2 \times \epsilon_{k, l}(m, n)$ is heuristically adopted, we show that (by comparing those results obtained using $\epsilon_{k, l}(m, n)=0$ and $\left.\epsilon_{k, l}(m, n) \neq 0\right)$ the BERs can be further increased and the PSNRs are moderate.

Under the circumstance that the proposed near-perfect cover data recovery algorithm is used, we are confident based on Table II that even the so-called "optimal watermark detector" [2], [5], [11], [27] is difficult to detect the survived (but few) watermark bits to sufficiently claim the existence of a watermark; otherwise, false positive probability is easy to occur. This validates our claim that efficient elimination of previously added watermark energy is indispensable to really remove the hidden watermark.

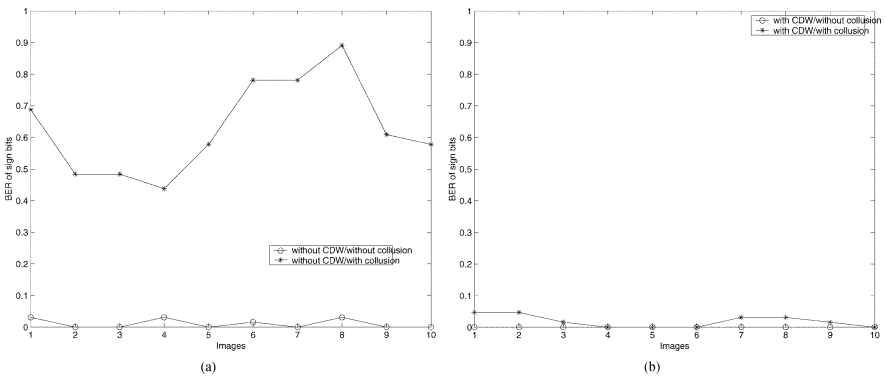

Fig. 5. Scenario 1 (BER of the estimated watermark's sign bits from an owner's perspective). (a) Method I; without using the CDW, most of the watermark's sign bits are correctly estimated using a collusion attack. (b) Method II; when the CDW is introduced, the watermark's sign bits mostly remain unchanged. This experiment confirms that the CDW is efficient in randomizing watermarks in order to disable collusion.

\section{B. CDW Resistance to Collusion Attack}

The collusion attack (operated by colluding $|\mathcal{C}|=|\Omega|=256$ blocks) was applied to Method I and Method II, respectively, on ten cover images. The resistance of the CDW to the collusion attack will be examined with respect to three scenarios: (s1) the BER of the estimated watermark's sign bits; (s2) the quality of a colluded image; and (s3) watermark detection after performing collusion.

As for (s1), Fig. 5(a) shows that when the CDW was not involved in watermark embedding, most watermark's sign bits were correctly estimated using the collusion attack. However, once the CDW was used, Fig. 5(b) shows that an owner could extract most of the watermark's sign bits no matter whether collusion was conducted or not. This experiment confirms that the CDW is able to prevent most watermark's sign bits from being correctly estimated under collusion.

Here, we simply use denoising for blind watermark detection (which is usually not optimal) to demonstrate the benefit of CDW. One may argue that this does not imply other techniques 
TABLE III

Scenario 3 (Watermark Detection Under Collusion): STATistical Comparison of BeR's Distributions. In THIS MEAN AND VARIANCE OF A COLLUDED IMAGE’s DistRIBUTION OF BERS ARE CALCULATED

\begin{tabular}{|c|c|c|c|c|c|c|c|c|c|c|}
\hline $\begin{array}{l}\text { Colluded image } \\
(\text { Method I }(|\mathcal{C}|=256)) \\
\text { mean } \\
\text { variance }\end{array}$ & $\begin{array}{l}\mathbf{X}_{\mathbf{1}}^{\mathbf{u}} \\
0.51 \\
0.006 \\
\end{array}$ & $\begin{array}{l}\mathbf{X}_{2}^{\mathbf{u}} \\
0.51 \\
0.004 \\
\end{array}$ & $\begin{array}{l}\mathbf{X}_{\mathbf{3}}^{\mathbf{u}} \\
0.50 \\
0.003 \\
\end{array}$ & $\begin{array}{l}0.50 \\
0.004 \\
\end{array}$ & $\begin{array}{l}\mathbf{X}_{\mathbf{5}}^{\mathbf{u}} \\
0.50 \\
0.004 \\
\end{array}$ & $\begin{array}{l}0.53 \\
0.008 \\
\end{array}$ & $\begin{array}{l}0.53 \\
0.005\end{array}$ & $\begin{array}{l}0.61 \\
0.015\end{array}$ & $\begin{array}{l}0.51 \\
0.005\end{array}$ & $\begin{array}{l}0.50 \\
0.004 \\
\end{array}$ \\
\hline $\begin{array}{l}\text { Colluded image } \\
(\text { Method II }(|\mathcal{C}|=256) \text { ) } \\
\text { mean } \\
\text { variance } \\
\text { Integrated BER } \\
\end{array}$ & $\begin{array}{l}\mathbf{X}_{\mathbf{1}}^{\mathbf{u}} \\
0.36 \\
0.007 \\
0.047 \\
\end{array}$ & $\begin{array}{l}\mathbf{X}_{\mathbf{2}}^{\mathbf{u}} \\
0.39 \\
0.005 \\
0.000 \\
\end{array}$ & $\begin{array}{l}\mathbf{X}_{\mathbf{3}}^{\mathbf{u}} \\
0.38 \\
0.015 \\
0.016 \\
\end{array}$ & $\begin{array}{l}\mathbf{X}_{\mathbf{4}}^{\mathbf{u}} \\
0.44\end{array}$ & $\begin{array}{l}\mathbf{X}_{\mathbf{5}}^{\mathbf{u}} \\
0.42\end{array}$ & $\begin{array}{l}\mathbf{X}_{\mathbf{6}}^{\mathbf{u}} \\
0.38\end{array}$ & $\begin{array}{l}\mathbf{X}_{\mathbf{7}}^{\mathbf{u}} \\
0.36 \\
0.030 \\
0.031 \\
\end{array}$ & $\begin{array}{l}\mathbf{X}_{\mathbf{8}}^{\mathbf{u}} \\
0.35 \\
0.019 \\
0.031 \\
\end{array}$ & $\begin{array}{l}\mathbf{X}_{\mathbf{9}}^{\mathbf{u}} \\
0.41\end{array}$ & $\begin{array}{l}\mathbf{X}_{\mathbf{1 0}}^{\mathbf{u}} \\
0.39 \\
0.017 \\
0.000 \\
\end{array}$ \\
\hline $\begin{array}{l}\text { Colluded image } \\
(\text { Method II }(|\mathcal{C}|=1)) \\
\text { mean } \\
\text { variance } \\
\text { Integrated BER }\end{array}$ & $\begin{array}{l}\mathbf{X}_{\mathbf{1}}^{\mathbf{u}} \\
0.47 \\
0.004 \\
0.266\end{array}$ & $\begin{array}{l}\mathbf{X}_{\mathbf{2}}^{\mathbf{u}} \\
0.48 \\
0.003 \\
0.203\end{array}$ & $\begin{array}{l}\mathbf{X}_{\mathbf{3}}^{\mathbf{u}} \\
0.48 \\
0.004 \\
0.172\end{array}$ & $\begin{array}{l}0.49 \\
0.003 \\
0.297\end{array}$ & $\begin{array}{l}0.48 \\
0.003 \\
0.234\end{array}$ & $\begin{array}{l}\mathbf{X}_{6}^{\mathbf{u}} \\
0.48 \\
0.004 \\
0.141\end{array}$ & $\begin{array}{l}\mathbf{X}_{\mathbf{7}}^{\mathbf{u}} \\
\\
0.47 \\
0.005 \\
0.234\end{array}$ & $\begin{array}{l}\mathbf{X}_{\mathbf{8}}^{\mathbf{u}} \\
\\
0.48 \\
0.005 \\
0.172\end{array}$ & $\begin{array}{l}0.48 \\
0.004 \\
0.219\end{array}$ & $\begin{array}{l}0.48 \\
0.003 \\
0.297\end{array}$ \\
\hline
\end{tabular}
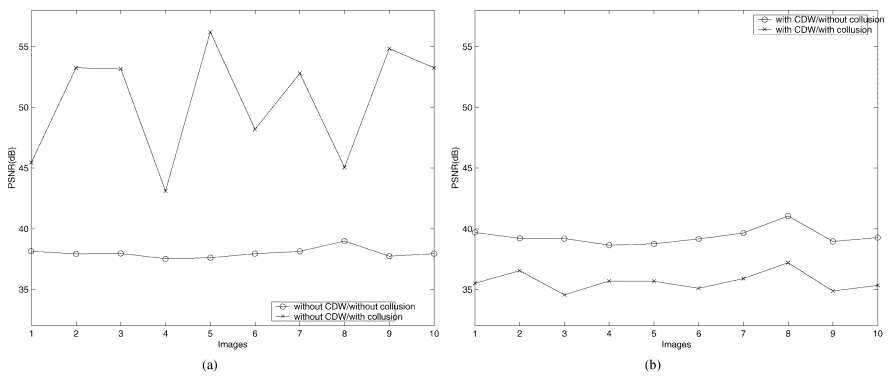

Fig. 6. Scenario 2 (quality of a colluded image). (a) Method I; without using the CDW, the PSNR values of the colluded images are higher than those of the watermarked images. (b) Method II; when the CDW is applied, the PSNR values of the colluded images are lower than those of the watermarked images. This experiment reveals that a collusion attack will fail to improve the fidelity of a colluded image when the CDW is involved.

(e.g., optimal watermark detectors) cannot detect the existence of a watermark. However, as we have shown in Table II that most of the watermark values were deleted, it is straightforward to know that under sufficiently small false positive even optimal detectors cannot guarantee to succeed.

As for (s2), the collusion attack (i.e., with all $\epsilon_{k, l}(m, n)$ 's equal to zero) is considered. It can be found in Fig. 6(a) that collusion improved the quality of the colluded images in terms of PSNR. However, the CDW could force collusion to undesirably degrade the quality of the colluded images, as shown in Fig. 6(b). This experiment demonstrates that the CDW is efficient in prohibiting a collusion attack from achieving efficient cover data recovery (5).

As for (s3), the collusion attack was performed using $|\mathcal{C}|=$ 256 since each image was decomposed into 256 blocks. The results of watermark detection measured in terms of BER between the original watermark and the extracted watermark are shown in Table III. For each image, 256 BER values were obtained. The statistical feature, i.e., mean and variance of BER values for each image was calculated. In Table III, when CDW was not employed, the mean BER for all colluded images approximate 0.5 and the variances are very small (the first row). Once the CDW was involved in embedding, detection results obtained from colluded images show that the mean BERs for all colluded images are almost lower than 0.4 (the second row). On the other hand, as Proposition 2 shows, the degradation of the CDW's resistance to collusion is lower bounded by the degener- ated collusion attack (i.e., with $|\mathcal{C}|=1$ ). We show the detection results with respect to $|\mathcal{C}|=1$ in the third row of Table III. By comparing the last two rows, it is observed that the mean BERs obtained using $|\mathcal{C}|=1$ are indeed larger than those obtained using $|\mathcal{C}|=256$, which is exactly consistent with our analysis.

In particular, if the watermarks extracted from all the image blocks were integrated to reconstruct the hidden watermark (i.e., collusion estimation), we can find that the integrated watermark obtained using Method II was close to its original. ${ }^{8}$ In fact, we find that the integrated BER values are significantly reduced (with $|\mathcal{C}|=256$ ) and are slightly reduced (with $|\mathcal{C}|=1$ ), as shown in Table III. However, the integrated results obtained using Method I still approximate 0.5 and fail to lower the BERs. These results indicate that the use of the CDW is able to keep lower BERs when a collusion attack is encountered. That's the reason why some works (e.g., [22], [31]) have proposed the embedding of multiple watermarks to enhance robustness.

In summary, as long as a media hash is involved in the construction of a watermark, the quality of the colluded images will be degraded, but it is still possible for the watermarks to be detected, as evidenced in Table III. Therefore, the merits of the CDW in helping multiple redundant watermark embedding methods to resist collusion have been thoroughly demonstrated.

\section{CDW Embedding versus False Positive}

As shown in Table III, collusion estimation of the watermark using Method II generates very low BERs. Now, we conduct an experiment to verify whether the false positive problem occurs when our CDW is embedded into an unwatermarked image. Table IV depicts the detection results in terms of BERs with respect to the ten cover images shown in Fig. 4. It is observed that the obtained BERs are nearly approximate to 0.5 , which demonstrates that the proposed collusion estimation-based watermark extraction is immune to false positive.

\section{CDW Resistance to the Copy Attack}

The copy attack was applied to Method I and Method II for comparison. One of the ten images was first watermarked, and

\footnotetext{
${ }^{8}$ Here, we aim to show the ability of the CDW in resisting the collusion attack and only simple denoising-based blind watermark extraction is used. Other sophisticated watermark detectors or error correcting codes could also be employed.
} 
TABLE IV

VALIDATION OF OUR CONTENT-DEPENDENT WATERMARK W.R.T. FALSE POSITIVE

\begin{tabular}{l||l|l|l|l|l|l|l|l|l|l}
\hline Cover image & $\mathbf{X}_{\mathbf{1}}$ & $\mathbf{X}_{\mathbf{2}}$ & $\mathbf{X}_{\mathbf{3}}$ & $\mathbf{X}_{\mathbf{4}}$ & $\mathbf{X}_{\mathbf{5}}$ & $\mathbf{X}_{\mathbf{6}}$ & $\mathbf{X}_{\mathbf{7}}$ & $\mathbf{X}_{\mathbf{8}}$ & $\mathbf{X}_{\mathbf{9}}$ & $\mathbf{X}_{\mathbf{1 0}}$ \\
\hline BER & 0.532 & 0.488 & 0.492 & 0.488 & 0.492 & 0.548 & 0.489 & 0.496 & 0.537 & 0.523 \\
\hline
\end{tabular}

then the watermark was estimated and copied to the other nine unwatermarked images to form nine counterfeit watermarked images. By repeating the above procedure, a total of 90 counterfeit watermarked images were obtained. The PSNR values of the 90 attacked images were in the range of $26 \sim 36 \mathrm{~dB}$ (no perceptual masking was considered). Our other goal was to verify whether a strong watermark's energy could defeat the CDW. As described previously, "collusion estimation" can yield more accurate detection results. Therefore, the results reported here were obtained using collusion estimation, i.e., all block-based detection results in an image are integrated as the final one. By applying the copy attack to Method I, the obtained 90 BER values fell within the interval [0.130 0.263]. However, when the CDW was introduced, these BERs increased significantly to the interval [0.468 0.549]. The experimental results are consistent with (22). Obviously, the proposed CDW is able to deter the detection of copied watermarks since the resultant BERs are significantly increased (again, we did not set a fixed threshold to determine the existence of a watermark since it may depend on many factors).

\section{CONCLUSIONS AND FUTURE WORK}

Although multiple watermarks can be embedded into an image to withstand geometrical distortions, we find that they are unfortunately vulnerable to collusion and copy attacks, and the desired geometric invariance is lost. In particular, we have proposed an efficient watermark estimation and recovery algorithm (which is regarded as an exquisite collusion attack) that can eliminate almost all watermark values. To cope with these problems, an anti-disclosure watermark with resistance to the watermark-estimation attack (WEA) has been investigated in this paper. Notably, we have pointed out analytically and experimentally that both accurate estimation of a watermark's sign and complete subtraction of a watermark's energy constitute the sufficient and necessary conditions for achieving effective watermark removal. As a consequence, we have introduced the concept of the media hash and combined it with hidden information to create the so-called content-dependent watermark (CDW), which can be used to prevent unauthorized watermark estimation. The characteristics of the CDW have been analyzed to verify its resistance to WEA. Finally, we would like to bring up a challenging problem, namely, that the "estimation+geometry" attack must be considered to evaluate the robustness of image watermarking methods.

The proposed image hash in its current status is sensitive to geometric distortions and could affect the resistance of CDW to them. In particular, when the image hash is combined with the block-based image watermarking scheme [44], the periodically regular watermark pattern disappears because the content-dependent watermarks resulting from all the image blocks look dissimilar such that the preset periodical regularity is destroyed. Therefore, we have further studied the geometrical invariance of image hashing in [24]. In our recent paper [25], the proposed CDW has been combined with geometric-invariant image hash to obtain a mesh-based content-dependent watermarking scheme that can resist both the geometric and estimation attacks.

\section{APPENDIX \\ CONFIDENCE IN WATERMARK Sign ESTIMATION UNDER COLLUSION}

Here, we will justify our confidence in collusive estimation of a watermark's sign using binomial probability distribution. Suppose each $\operatorname{sgn}\left(W_{b}^{e}(i)\right), b \in \mathcal{C}$ is regarded as a trial, and that the trials are independent. This kind of trial will result in one of two possible outcomes: +1 and -1 . Each outcome will occur with equal probability: 0.5 . Now, our confidence in the occurrence of $\operatorname{sgn}\left(W_{b}^{e}(i)\right)$ is formulated as the probability $P_{s}$ of $\operatorname{sgn}\left(W_{b}^{e}(i)\right)$ 's, which are observed in $|\mathcal{C}|$ samples, $\Delta=\left\{\operatorname{sgn}\left(W_{1}^{e}(i)\right), \operatorname{sgn}\left(W_{2}^{e}(i)\right), \ldots, \operatorname{sgn}\left(W_{|\mathcal{C}|}^{e}(i)\right)\right\}$. Let $\beta$ be the random variable denoting the number of $\operatorname{sgn}\left(W_{b}^{e}(i)\right)$ observed in $\Delta$. As a consequence, $P_{s}$ can be expressed as

$$
P_{s}\left(\beta>\frac{|\mathcal{C}|}{2}\right)=\sum_{n=|\mathcal{C}| / 2+1}^{|\mathcal{C}|}\left(\begin{array}{c}
|\mathcal{C}| \\
n
\end{array}\right) 0.5^{|\mathcal{C}|} \text {. }
$$

Looking at the table of binomial probabilities [26, pp. 685-688], we can find that $P_{s}$ will increase rapidly (usually it will be larger than 0.8 ) as long as $n$ is slightly larger than $|\mathcal{C}| / 2$. The larger $P_{s}$ is, the more confident we are. Hence, we have sufficient confidence to rely on the collusion attack to determine the sign of a hidden watermark.

\section{ACKNOWLEDGMENT}

The authors thank the anonymous reviewers for their valuable comments in improving this paper.

\section{REFERENCES}

[1] M. Barni, F. Bartolini, V. Cappellini, and A. Piva, "Copyright protection of digital images by embedded unperceivable marks," Image Vis. Comput., vol. 16, pp. 897-906, 1998.

[2] M. Barni, F. Bartolini, A. E. Rosa, and A. Piva, "A new decoder for the optimum recovery of nonadditive watermarks," IEEE Trans. Image Process., vol. 10, no. 5, pp. 755-766, May 2001.

[3] J. Barr, B. Bradley, and B. T. Hannigan, "Using digital watermarks with image signatures to mitigate the threat of the copy attack," in Proc. IEEE Int. Conf. Acoust., Speech, Signal Process., 2003, vol. III, pp. 69-72.

[4] P. Bas, J. M. Chassery, and B. Macq, "Geometrically invariant watermarking using feature points," IEEE Trans. Image Process., vol. 11, no. 9, pp. 1014-1028, Sep. 2002.

[5] Q. Cheng and T. S. Huang, "Robust optimum detection of transform domain multiplicative watermarks," IEEE Trans. Signal Process., vol. 51, no. 4, pp. 906-924, 2003.

[6] I. J. Cox, M. L. Miller, and J. A. Bloom, Digital Watermarking. San Mateo, CA: Morgan Kaufmann, 2002.

[7] S. Craver, N. Memon, B. L. Yeo, and M. M. Yeng, "Resolving rightful ownership with invisible watermarking techniques: Limitations, attacks, and implications," IEEE J. Sel. Areas Commun., vol. 16, no. 4, pp. 573-586, May 1998. 
[8] F. Deguillaume, S. Voloshynovskiy, and T. Pun, "Secure hybrid robust watermarking resistant against tampering and copy attack," Signal Process., vol. 83, 2003.

[9] R. Dursternfeld, "Algorithm 235: Random permutation [G6]," Commun. ACM, pp. 420-420, 1964.

[10] J. Fridrich, "Visual hash for oblivious watermarking," in Proc. SPIE: Security and Watermarking of Multimedia Contents II, 2000, vol. 3971.

[11] J. Hernandez, M. Amado, and F. Perez-Gonzalez, "DCT-domain watermarking techniques for still images: Detector performance analysis and a new structure," IEEE Trans. Image Process., vol. 9, no. 1, pp. 55-68, Jan. 2000.

[12] IEEE J. Sel. Areas Commun.: Special Issue on Copyright and Privacy Protection, vol. 16, no. 4, 1998.

[13] IEEE Trans. Signal Process.: Special Issue on Signal Processing for Data Hiding in Digital Media and Secure Content Delivery, vol. 51, no. $4,2003$.

[14] T. Kalker, G. Depovere, J. Haitsma, and M. Maes, "A video watermarking system for broadcast monitoring," in Proc. SPIE, 1999, vol. 3657, pp. 103-112.

[15] D. Kirovski, H. S. Malvar, and Y. Yacobi, "A dual watermarking and fingerprinting system," IEEE Multimedia, vol. 11, no. 3, pp. 59-73, Jul.-Sep. 2004.

[16] D. Knuth, The Art of Computer Programming, 3rd ed. Reading, MA: Addison-Wesley, 1997, vol. 2.

[17] M. Kutter, S. Voloshynovskiy, and A. Herrigel, "The watermark copy attack," in Proc. SPIE: Security and Watermarking of Multimedia Contents II, 2000, vol. 3971

[18] J. S. Lee, "Digital image enhancement and noise filtering by use of local statistics," IEEE Trans. Pattern Anal. Machine Intell., vol. 2, no. 2, pp. 165-168, Mar. 1980.

[19] C. Y. Lin and S. F. Chang, "A robust image authentication method distinguishing JPEG compression from malicious manipulation," IEEE Trans. Circuits Syst. Video Technol., vol. 11, no. 2, pp. 153-168, Feb. 2001.

[20] C. S. Lu, H. Y. Liao, and M. Kutter, "Denoising and copy attacks resilient watermarking by exploiting prior knowledge at detector," IEEE Trans. Image Process., vol. 11, no. 3, pp. 280-292, Mar. 2002.

[21] C. S. Lu and H. Y. M. Liao, "Structural digital signature for image authentication: An incidental distortion resistant scheme," IEEE Trans. Multimedia, vol. 5, no. 2, pp. 161-173, Jun. 2003.

[22] C. S. Lu and C. Y. Hsu, "Content-dependent anti-disclosure image watermark," in Proc. 2nd Int. Workshop Digital Watermarking (IWDW), Seoul, Korea, 2003, LNCS 2939, pp. 61-76.

[23] C. S. Lu and C. M. Yu, "On the security of mesh-based media hashdependent watermarking against protocol attacks," in Proc. IEEE Int. Conf. Multimedia Expo., Amsterdam, The Netherlands, Jul. 2005, pp. $169-172$.

[24] C. S. Lu and C. Y. Hsu, "Geometric distortion-resilient image hashing scheme and its applications on copy detection and authentication," ACM Multimedia Systems J., Special Issue on Multimedia and Security, vol. 11, no. 2, pp. 159-173, 2005.

[25] C. S. Lu, S. W. Sun, C. Y. Hsu, and P. C. Chang, "Media hash-dependent image watermarking resilient against both geometric attacks and estimation attacks based on false positive-oriented detection," IEEE Trans. Multimedia, vol. 8, no. 4, pp. 668-685, Aug. 2006.

[26] W. Mendenhall, R. L. Scheaffer, and D. D. Wackerly, Mathematical Statistica with Applications. Boston, MA: Duxbury, 1986.

[27] A. Nikolaidis and I. Pitas, "Asymptotically optimal detection for additive watermarking in the DCT and DWT domains," IEEE Trans. Image Process., vol. 12, no. 5, pp. 563-571, May 2003.

[28] "Special Session on Media Recognition," in Proc. IEEE Int. Workshop Multimedia Signal Processing, St. Thomas, U.S. Virgin Islands, Dec. 2002.

[29] "Special Session on Media Identification," in Proc. IEEE Int. Conf. Multimedia Expo, Taipei, Taiwan, R.O.C., 2004.

[30] Proc. IEEE, vol. 87, 1999

[31] M. Ramkumar and A. N. Akansu, "A robust scheme for oblivious detection of watermarks/data hiding in still images," in Proc. SPIE Multimedia Systems and Applications, 1998, vol. 3528, pp. 474-481.

[32] J. S. Seo, J. Haitsma, T. Kalker, and C. D. Yoo, "A robust image fingerprinting system using the radon transform," Signal Process.: Image Commun., vol. 19, pp. 325-339, 2004.

[33] Signal Process.: Special Issue on Information Theoretic Aspects of Digital Watermarking, vol. 81, no. 6, 2001

[34] J. K. Su and B. Girod, "Power-spectrum condition for energy-efficient watermarking," IEEE Trans. Multimedia, vol. 4, no. 4, pp. 539-560, Dec. 2002
[35] K. Su, D. Kundur, and D. Hatzinakos, "Statistical invisibility for collusion-resistant digital video watermarking," IEEE Trans. Multimedia, vol. 7, no. 1, pp. 43-51, Feb. 2005.

[36] Q. Sun and S. F. Chang, "A secure and robust digital signature scheme for JPEG2000 image authentication," IEEE Trans. Multimedia, vol. 7, no. 3, pp. 480-494, Jun. 2005.

[37] M. D. Swanson, B. Zhu, and A. H. Tewfik, "Multiresolution scene-based video watermarking using perceptual models," IEEE J. Sel. Areas Commun., vol. 16, no. 4, pp. 540-550, May 1998.

[38] C. W. Tang and H. M. Hang, "A feature-based robust digital image watermarking scheme," IEEE Trans. Signal Process., vol. 51, no. 4, pp. 950-959, Apr. 2003

[39] W. Trappe, M. Wu, and K. J. R. Liu, "Collusion-resistant fingerprinting for multimedia," in Proc. IEEE Int. Conf. Acoust., Speech, Signal Process., Orlando, FL, May 2002, vol. 4, pp. 3309-3312.

[40] W. Trappe, M. Wu, J. Wang, and K. J. R. Liu, "Anti-collusion fingerprinting for multimedia," IEEE Trans. Signal Process., vol. 51, no. 4, pp. 1069-1087, Apr. 2003.

[41] S. Voloshynovskiy, A. Herrigel, N. Baumgartner, and T. Pun, "A stochastic approach to content adaptive digital image watermarking," in Proc. 3rd Int. Workshop Information Hiding, Dresden, Germany, 1999 , LNCS 1768, pp. 211-236.

[42] S. Voloshynovskiy, S. Pereira, A. Herrigel, N. Baumgartner, and T. Pun, "Generalized watermarking attack based on watermark estimation and perceptual remodulation," in Proc. SPIE: Security and Watermarking of Multimedia Contents II, San Jose, CA, 2000, vol. 3971.

[43] S. Voloshynovskiy, S. Pereira, V. Iquise, and T. Pun, "Attack modelling: Towards a second generation watermarking benchmark," Signal Process., vol. 81, no. 6, pp. 1177-1214, 2001.

[44] S. Voloshynovskiy, F. Deguillaume, and T. Pun, "Multibit digital watermarking robust against local nonlinear geometrical distortions," in Proc. IEEE Int. Conf. Image Process., 2001, pp. 999-1002.

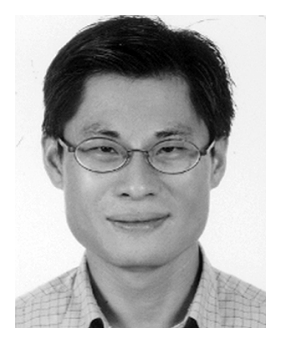

Chun-Shien Lu (M'99) received the Ph.D. degree in electrical engineering from National Cheng-Kung University, Tainan, Taiwan, R.O.C., in 1998.

From October 1998 to July 2002, he was with the Institute of Information Science, Academia Sinica, Taiwan, as a Postdoctoral Fellow for his military service. From August 2002 to June 2006, he was an Assistant Research Fellow at the same institute. Since July 2006, he has been an Associate Research Fellow. He is the editor of the book Multimedia Security: Steganography and Digital Watermarking Techniques for Protection of Intellectual Property (Idea Group, January 2004). His current research interests mainly focus on various topics (including security, networking, and signal processing) of multimedia and time-frequency analysis of signals.

Dr. Lu organized a special session on Multimedia Security in the 2nd and 3rd IEEE Pacific-Rim Conference on Multimedia, respectively (2001 and 2002). He co-organized two special sessions (in the area of media identification and DRM) in the 5th IEEE International Conference on Multimedia and Expo (ICME), 2004. He was a guest co-editor of EURASIP Journal on Applied Signal Processing, special issue on Visual Sensor Network in 2005. He holds two U.S. patents, two R.O.C. patents, and one Canadian patent in digital watermarking. He has received the paper awards many times from the Image Processing and Pattern Recognition society of Taiwan for his work on data hiding. He was a co-recipient of a National Invention and Creation Award in 2004. He is a member of the IEEE and ACM.

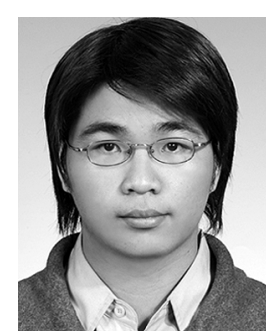

Chao-Yung Hsu has been a Research Assistant in the Institute of Information Science, Academia Sinica, Taipei, Taiwan, R.O.C., since 2003 for his army service. Since September 2005, he has also been working toward the Ph.D. degree in the Graduate Institute of Communication Engineering of National Taiwan University. His research interests are in multimedia signal processing, data hiding, and halftoning. 